\title{
Research on the Dynamic Characteristics of the Crack Damage of the Seal-Rotor System
}

\section{Fengchao Huang ( $\nabla$ 878995859@qq.com )}

Shenyang University of Technology https://orcid.org/0000-0003-0148-4898

\section{Lipeng Wang}

Ningbo University of Technology

\section{Yuegang Luo}

Dalian Minzu University College of Mechanical and Electronic Engineering

Changzhen Chen

Shenyang University of Technology

\section{Research Article}

Keywords: labyrinth seal, Crack damage,Airflow excitation, finite element method, Crack parameters

Posted Date: March 1st, 2022

DOI: https://doi.org/10.21203/rs.3.rs-1372175/v1

License: (9) This work is licensed under a Creative Commons Attribution 4.0 International License. Read Full License 


\title{
Research on the Dynamic Characteristics of the Crack Damage of the Seal-Rotor System
}

\author{
Lipeng Wang ${ }^{\mathrm{a}}$, Fengchao Huang ${ }^{\mathrm{c}},{ }^{*}$, Yuegang Luo $^{\mathrm{b}}$, Changzhen Chen ${ }^{\mathrm{c}}$
}

(a.School of Mechanical Engineering, Ningbo University of Technology, Ningbo 315016, China

b. School of Mechanical and Electronic Engineering, Dalian Minzu University,Dalian 116600, PR China;

c. Shenyang University of Technology, School of Mechanical Engineering, Shenyang 110870,

$$
\text { Peoples R China) }
$$

\begin{abstract}
In the rotor system, the labyrinth seal system is the core component to suppress fluid leakage between the rotor and the stator. This paper analyzes the vibration characteristics of the coupling of air-induced vibration and crack fault. First, perform numerical simulation analysis and test verification on the response of the system with no sealing force and crack failure; Subsequently, the system with sealing force and different crack parameters was analyzed for numerical simulation analysis, and then the influence of crack damage failure on other sealing parameters (including sealing pressure difference, sealing gap, and sealing length) was studied; Finally, the influence of double crack damage (damage location, damage degree, phase difference angle) on the rotor system is analyzed. The results show that when the crack depth increases to a certain value, it will also cause the superharmonic resonance phenomenon in the subcritical speed region of the system; When the system has a sealing force, it is found that the airflow excitation frequency of the system can be affected as the degree of crack damage increases; The coupled dynamic response of airflow excitation and crack faults shows a rich spectrum of nonlinear phenomena, which is closely related to the degree of cracks and sealing parameters. Increasing the crack angle will weaken the impact of crack damage on the system. This research can provide a theoretical basis for detecting and diagnosing crack faults in the labyrinth seal rotor system.
\end{abstract}

Keywords:labyrinth seal, Crack damage,Airflow excitation, finite element method, Crack parameters.

Corresponding author.

Email address: 878995859@qq.com (F. C. Huang) 


\begin{tabular}{|c|c|}
\hline$A_{x}, A_{y}$ & The vibration displacement of the system in $X / Y$-direction. \\
\hline$A_{s}$ & Unit cross-sectional area. \\
\hline$A_{c e}$ & Remaining area of crack section. \\
\hline$C$ & Damping matrix of the rotor system. \\
\hline$c$ & Clearance of seal. \\
\hline$D$ & Gyro matrix of the rotor system. \\
\hline$D_{d}^{m}$ & Disc gyro matrix at the m node. \\
\hline $\boldsymbol{D}_{s}$ & Rotary shaft gyro matrix of system. \\
\hline $\boldsymbol{D}_{d}$ & Disk gyro matrix of the system . \\
\hline$D_{f}$ & Equivalent damping in sealing force. \\
\hline$E$ & Young's modulus of rotor material. \\
\hline$e$ & Relative eccentricity of the rotor. \\
\hline $\boldsymbol{F}_{p}$ & Eccentric force vector. \\
\hline $\boldsymbol{F}_{f}$ & Sealing force vector. \\
\hline$f(t)$ & Crack opening and closing function. \\
\hline$F_{f x}, F_{f y}$ & Component force of sealing force in $x$ and $y$ directions. \\
\hline$f_{1}, f_{2}$ & The first and second natural frequencies of the rotor system. \\
\hline$f_{f}$ & Airflow excitation frequency. \\
\hline $\boldsymbol{G}$ & Gravity vector. \\
\hline$G_{s}$ & Shear modulus. \\
\hline $\boldsymbol{G}_{n}$ & Gyro Matrix of Shaft Unit. \\
\hline$h$ & Crack depth. \\
\hline$h_{m}$ & The thickness of the disc. \\
\hline I & Moment of inertia of section. \\
\hline$J_{d}$ & Diameter moment of inertia. \\
\hline$J_{p}$ & Polar moment of inertia. \\
\hline $\boldsymbol{K}$ & Stiffness matrix of the rotor system. \\
\hline$K_{f}$ & Equivalent stiffness of sealing force. \\
\hline $\boldsymbol{K}_{n}$ & Stiffness matrix of shaft element. \\
\hline $\boldsymbol{K}_{s}$ & Stiffness matrix of the system shaft. \\
\hline $\boldsymbol{K}^{l \boldsymbol{w}}{ }_{c}$ & The matrix of the stiffness reduction of the crack element. \\
\hline$L$ & The radius of the sealing disc. \\
\hline$l$ & Unit length of shaft segment. \\
\hline$l_{m}$ & Sealing length. \\
\hline$M$ & The mass matrix of the rotor system. \\
\hline $\boldsymbol{M}_{n}$ & Mass matrix of shaft element. \\
\hline $\boldsymbol{M}_{s}$ & The mass matrix of the system shaft. \\
\hline $\boldsymbol{M}_{d}^{m}$ & Disc mass matrix at the m-th node. \\
\hline $\boldsymbol{M}_{d}$ & Mass matrix of the system disc. \\
\hline$m$ & Position number of disc node. \\
\hline$m_{d}$ & The quality of the disc. \\
\hline$m_{f}$ & Equivalent mass in sealing force. \\
\hline me & Eccentricity of unbalance mass of the seal disk. \\
\hline$n$ & Shaft segment number. \\
\hline$Q$ & The resultant external force vector of the rotor system. \\
\hline$R$ & The radius of the sealing disc. \\
\hline
\end{tabular}




\begin{tabular}{ll}
\hline$R_{m}$ & Sealing radius. \\
$R_{a}$ & Reynolds number of axial flow. \\
$R_{v}$ & Reynolds Number of Circumferential Flow. \\
$r_{g}$ & Radius of gyration. \\
$r_{m}$ & The radius of the disc. \\
$\boldsymbol{u}_{n}$ & Displacement vector of the nth axis segment. \\
$v$ & Poisson's ratio of rotor material. \\
$v_{a}$ & Axial velocity of air flow. \\
$z$ & Inlet loss coefficient. \\
$\alpha$ & Crack angle. \\
$\rho$ & Material density of the rotor. \\
$\phi$ & Transverse shear parameters. \\
$\omega$ & Rotating angular velocity of rotor. \\
$\vartheta$ & Shear factor. \\
$\xi_{1}, \xi_{2}$ & Rotor system first and second modal damping ratio. \\
$\tau$ & Average flow velocity ratio in the circumferential direction of the fluid. \\
$\sigma$ & Friction loss gradient coefficient. \\
$\Delta P$ & Sealing inlet and outlet pressure difference. \\
$\gamma$ & Dimensionless crack depth. \\
$\tau \omega$ & Average flow rate ratio in the sealed cavity. \\
$\mu$ & Crack depth ratio. \\
\hline
\end{tabular}

\section{Introduction}

The labyrinth seal is an effective sealing structure widely used between the shaft end and various levels in modern aero-engines, compressors, steam turbines and other power mechanical structures. It has a simple structure, no friction, low power consumption, long service life, and no need for lubrication and maintenance. Convenience and other characteristics, its function is to suppress the fluid leakage between the stator and the rotor, thereby reducing the leakage loss[1]. Since the work requires the sealed shaft to maintain high-speed rotation for a long time, the flexible characteristics of the rotor itself will cause the shaft to be biased in the mechanical device so that the air pressure distribution in the sealed cavity does not correspond to the gap of the seal, and the air pressure distribution is uneven. The rotor produces self-excited vibration, and if this phenomenon occurs, it will cause significant damage to the mechanical equipment. Muzynska once pointed out: Rubbing and cracking are usually easy to occur in the seal[2].In fact, due to installation error, fatigue loss, external force impact, etc., the crack failure of the seal-rotor system is very easy to occur. If it can not be found and handled in time, other failures will cause secondary damage to the equipment. Therefore, to improve the operating stability and safety of this type of system, it is of great significance to study the rotor system crack failure's complex dynamic characteristics under airflow excitation force and analyze the influence of some essential factors parameters on the system.

sealing force model and the study of dynamic characteristics. In terms of models, it can be divided into 
parametric analytical models and numerical calculation models of the sealing force. The parametric analytical models include the Thomas-Alford and Muzynska models, while the numerical calculation models are mainly based on the control body models, such as Wang et al.[3]through the application of a single control body model and perturbation method to dynamic modelling and calculation of the rotor system with a labyrinth seal. In recent years, more and more scholars have adopted computational fluid dynamics (CFD) methods to study flow field problems in seals. For example, Cangioli F et al. [4] proposed a new labyrinth seal thermoelastic body flow model, taking the cross-tooth labyrinth seal of a steam turbine as the research object, considering different pressures, speeds, and inlet pre-rotation ratios. The influence of sealing stability. There is also Kirk et al.[5,6] using CFX-TASCflow fluid dynamics software to solve and study its influencing factors. Avza C et al.[7]proposed a transient CFD method to calculate the nonlinear dynamic characteristics of the coupled rotor seal system on this basis.

In terms of dynamic characteristics research, one is to improve the stability of the sealing system by solving the dynamic characteristic coefficients and studying the influencing factors of cross stiffness and primary damping. For example, Subramanian S et al. [8] considered adding centrifugal to the rotor dynamics of the turbine seal of a gas turbine and carried out a numerical study, and found that under the relatively low-pressure ratio combination, a relatively low-pressure ratio and high speed, increasing centrifugation is not conducive to system stability. The second is to study the influencing factors of seal leakage to achieve the purpose of reducing leakage. For example, Sun Dan et al. [9] studied the influence of anti-swirling flow on the static and dynamic characteristics of the seal from both theoretical and experimental aspects; Liu Xingwang et al.[10]used the macro-energy method and the viscous flow theory to calculate the leakage in the radial labyrinth seal of the scroll compressor. The third is to study non-linear vibration problems, including air flow-induced seal vibration, or coupling failure factors such as oil film instability, rubbing, and cracking. For example, Ma W et al.[11] studied the influence of seal length and diameter on system dynamics; Shyu SH et al. [12] applied the finite element method to model the seal-rotor system with rolling bearing support and analyzed the influence of the geometric parameters of the seal structure on the seal-bearing-rotor system.

In terms of the research on the vibration characteristics and mechanism of crack damage in the rotor system, Rao Xiaobo et al. [13] studied the cracked rotor system with an oil film and found a chaotic "eye" phenomenon in the dynamic characteristics, which is helpful for the control and diagnosis of crack failure in the rotor system. Darpe et al. $[14,15]$ simulated the two-way coupled crack model with the intensity factor zero method, studied the effect of the crack on the transient vibration of the Jeffcott rotor and analyzed the unbalanced response of the system. Patel et al. $[16,17]$ established a finite element model of a rotor system with oblique cracks based on the theory of fracture mechanics. They studied the effect of the crack azimuth on the stiffness and the vibration response characteristics of the system. T Szolc, P et al.[18]established a cracked rotor finite element model using fracture mechanics theory, 
simulated the crack breathing effect with a cosine model, and mainly analyzed the response characteristics of $1 / 3$ times the critical speed of the system under different double crack parameters. Lu Zhenyong et al. [19] studied the effect of crack depth and crack location on the amplitude, and reached important conclusions. [20,21]established the finite element equation of an asymmetric rotor and studied the influence of transverse breathing cracks on the asymmetric rotor-bearing system.

There are also many achievements in the research on the coupling of crack faults and other faults: Palacios-Pineda Luis M. et al. [22] took the Jeffcott rotor system as the research object, considered the influence of transverse crack on system vibration, and obtained relevant conclusions through simulation calculation and experimental verification. Luo Yuegang et al.[23]established the dynamic modelling of a double-span rotor system with a coupling failure of cracks and support loosening by applying the concentrated mass method, and concluded that the loosening loss has a more significant impact on the system when the coupling failure occurs. Yang Yongfeng et al. [24]studied the rotor system with loosening and crack coupling faults and used the centralized mass method to model the system, and the crack switch model used the cosine model method. Based on the single-span single-disk rotor system considering the effect of oil film force. Xiang Ling et al.[25] analyzed the nonlinear behaviour of the rotor system coupled with multiple faults, and obtained the coupling effects of cracks, rubbing and oil film instability through experiments and simulation calculations.

Judging from the existing literature, the current research results mostly use the Jeffcott rotor as the research object for modelling and analysis and focus on considering single factors such as air-induced vibration or crack failure. The main research methods for the coupled faults containing cracks are the vibration characteristics of crack-oil film instability, crack looseness and crack-rubbing. However, there are relatively few studies on the crack failures that are most likely to occur in the seal-rotor system, which considers airflow excitation factors. It provides a specific theoretical basis for revealing the mechanism of the influence of the crack fault on the fluid excitation and the identification and diagnosis of the air flow-induced vibration-crack coupling fault in the seal-rotor system.

The structure of this article is: The second section establishes the dynamic equation of the labyrinth seal-rotor system, the finite element model of the rotor system, and the crack stiffness model of the damage breathing effect; The third section compares and analyzes the influence of crack damage on the nonlinear dynamic response of the system with or without air force in the system, and analyzes the influence of different crack damage degrees and damage locations on the system; In the fourth section, based on the research in the third section, the vibration response characteristics of the crack-damaged rotor system under the condition of changing the important sealing parameters of the seal-rotor system, and analyze the instability speed and the change law of the excitation frequency after the occurrence of airflow excitation; The fifth section analyzes the effect of double crack damage (damage location, damage degree, phase difference angle) on the rotor system. 


\section{The establishment of the seal-rotor system model}

\subsection{System dynamics model}

The finite element model of the rotor system is shown in Fig. 1(a). On the left is the disk with a sealing device with a radius of $\mathrm{R}=200 \mathrm{~mm}$ and a length of $\mathrm{L}=100 \mathrm{~mm}$. The rotating shaft adopts a stepped shaft, and there are two identical interleaved labyrinth seals on the disc. The length of the two seals is $15 \mathrm{~mm}$. The air inlet is located in the centre of the sealed stator. The system is divided into 27 shaft sections. The numbers in the figure indicate node numbers, totalling 28. The sealing disc is divided into 6-9, a total of 4 shaft sections, and the mass of the disc is added to the shaft section nodes. This paper uses the Timoshenko beam element to simulate the shaft element. As shown in Fig. 1(b), there are two section nodes $\mathrm{A}$ and $\mathrm{B}$ in the shaft segment, and each node has 6 degrees of freedom, which are translation and rotation along with the three directions of $\mathrm{x}, \mathrm{y}$, and $\mathrm{z}$, respectively, and the corresponding translation displacement and rotation angle. They are denoted as $x_{A}\left(x_{B}\right) 、 y_{A}\left(y_{B}\right) 、 z_{A}\left(z_{B}\right)$ and $\theta_{x A}\left(\theta_{x B}\right) 、 \theta_{y A}\left(\theta_{y B}\right) 、 \theta_{z A}\left(\theta_{z B}\right)$, respectively.Only the radial vibration is studied here, and the axial vibration is not considered, so the displacement in the z-axis direction and the rotation angle are ignored, then each node of the shaft element has 4 degrees of freedom, and the displacement vector of the nth shaft element is:

$$
\mathbf{u}_{n}=\left[x_{A}, y_{A}, \theta_{x A}, \theta_{v A}, x_{B}, y_{B}, \theta_{x B}, \theta_{y B}\right]^{\mathrm{T}}, n=1,2, \ldots \ldots, 27
$$

According to [26] the middle element mass matrix $\boldsymbol{M}_{n}$ of the Timoshenko beam is, the element stiffness matrix $\boldsymbol{K}_{n}$, the element gyro matrix $\boldsymbol{G}_{n}$, and the 27 element mass matrices $\boldsymbol{M}_{1}$ to $\boldsymbol{M}_{27}$ are assembled in the form of Fig. 2. The shaded parts in the figure indicate The matrix elements of the common nodes contained in two adjacent shaft segments can be obtained from the 112-dimensional shaft mass matrix $\boldsymbol{M}_{s}$. Similarly, the stiffness matrix $\boldsymbol{K}$ and the gyro matrix $\boldsymbol{D}_{s}$ can be obtained.

The unit mass matrix $\boldsymbol{M}_{n}$ is:

$$
\boldsymbol{M}_{n}=\rho A_{s} l\left[\begin{array}{cccccccc}
a & & & & & & & \\
0 & a & & & & & & \\
0 & -c & g & & & & & \\
c & 0 & 0 & g & & & & \\
b & 0 & 0 & d & a & & & \\
0 & b & -d & 0 & 0 & a & & \\
0 & d & f & 0 & 0 & c & g & \\
-d & 0 & 0 & f & -c & 0 & 0 & g
\end{array}\right]
$$

In the matrix, $a, b, c, d, f$ and $g$ are: 


$$
\begin{aligned}
& a=\frac{\frac{13}{35}+\frac{7}{10} \phi+\frac{1}{3} \phi^{2}+\frac{6}{5}\left(\frac{r_{g}}{l}\right)^{2}}{(1+\phi)^{2}} \\
& b=\frac{\frac{9}{70}+\frac{3}{10} \phi+\frac{1}{6} \phi^{2}-\frac{6}{5}\left(\frac{r_{g}}{l}\right)^{2}}{(1+\phi)^{2}} \\
& c=\frac{\left[\frac{11}{210}+\frac{11}{120} \phi+\frac{1}{24} \phi^{2}+\left(\frac{1}{10}-\frac{1}{2} \phi\right)\left(\frac{r_{g}}{l}\right)^{2}\right] l}{(1+\phi)^{2}} \\
& d=\frac{\left[\frac{13}{420}+\frac{3}{40} \phi+\frac{1}{24} \phi^{2}-\left(\frac{1}{10}-\frac{1}{2} \phi\right)\left(\frac{r_{g}}{l}\right)^{2}\right] l}{(1+\phi)^{2}} \\
& f=\frac{\left[\frac{1}{140}+\frac{1}{60} \phi+\frac{1}{240} \phi^{2}+\left(\frac{1}{30}+\frac{1}{6} \phi-\frac{1}{6} \phi^{2}\right)\left(\frac{r_{g}}{l}\right)^{2}\right] l^{2}}{(1+\phi)^{2}} \\
& g= \\
& {\left[\frac{1}{105}+\frac{1}{60} \phi+\frac{1}{120} \phi^{2}+\left(\frac{2}{15}+\frac{1}{6} \phi+\frac{1}{3} \phi^{2}\right)\left(\frac{r_{g}}{l}\right)^{2}\right] l^{2}} \\
& (1+\phi)^{2}
\end{aligned}
$$

Element stiffness matrix $\boldsymbol{K}_{n}$ :

$$
\boldsymbol{K}_{n}=\left[\begin{array}{cccccccc}
h & & & & & & & \\
0 & h & & & & & & \\
0 & -i & j & & & & & \\
i & 0 & 0 & j & & & & \\
-h & 0 & 0 & -i & h & & & \\
0 & -h & i & 0 & 0 & h & & \\
0 & -i & k & 0 & 0 & i & j & \\
i & 0 & 0 & k & -i & 0 & 0 & j
\end{array}\right]
$$

Where $h, i, j$ and $k$ are respectively:

$$
h=\frac{12 E I}{l^{3}(1+\phi)} \quad i=\frac{6 E I}{l^{2}(1+\phi)} \quad j=\frac{(4+\phi) E I}{l(1+\phi)} \quad k=\frac{(2-\phi) E I}{l(1+\phi)}
$$

Unit gyro matrix $\boldsymbol{G}_{n}$ :

$$
\boldsymbol{G}_{n}=2 \omega \rho A_{s} l\left[\begin{array}{ccccccccc}
0 & & & & & & & \\
-p & 0 & & & & & & \\
-q & 0 & 0 & & & & & \\
0 & -q & -s & 0 & & & & \\
0 & -p & -q & 0 & 0 & & & \\
p & 0 & 0 & -q & -p & 0 & & \\
-q & 0 & 0 & w & q & 0 & 0 & \\
0 & -q & -w & 0 & 0 & q & -s & 0
\end{array}\right]
$$

Where $p, q, s$ and $w$ are respectively: 


$$
\begin{aligned}
& p=\frac{\left(\frac{6}{5}\right) r_{g}^{2}}{l^{2}(1+\phi)^{2}} \\
& q=\frac{-\left(\frac{1}{10}-\frac{1}{2} \phi\right) r_{g}^{2}}{l^{2}(1+\phi)^{2}} \\
& s=\frac{\left(\frac{2}{15}+\frac{1}{6} \phi+\frac{1}{3} \phi^{2}\right) r_{g}^{2}}{(1+\phi)^{2}} \\
& w=\frac{-\left(\frac{1}{30}+\frac{1}{6} \phi-\frac{1}{6} \phi^{2}\right) r_{g}^{2}}{(1+\phi)^{2}}
\end{aligned}
$$

$E$ is Young's modulus of the material, $\rho$ is the material density, $A_{s}$ is the unit cross-sectional area, $l$ is the unit length, $I$ is the moment of inertia of the section, and $\omega$ is the rotational angular velocity of the rotor. In addition, the radius of gyration $r_{g}$ and the transverse shear parameter $\phi$ can be expressed by equation (8):

$$
r_{g}=\sqrt{\frac{I}{A_{s}}} \quad \phi=\frac{12 E I}{\vartheta A_{s} G_{s} l^{2}}
$$

Where $G_{s}$ is the shear modulus, $\vartheta$ is the shear coefficient, and the shear coefficient is expressed as: $v$ is the Poisson's ratio of the material, the solid shaft is: $\vartheta=\frac{6(1+v)}{(7+6 v)}$, the hollow shaft is: $\vartheta=\frac{2(1+v)}{(4+3 v)}$.

The mass of the disc of the system adopts a centralized mass model in this paper, and its mass matrix and gyro force matrix can be expressed as $\boldsymbol{D}_{\boldsymbol{d}}, \boldsymbol{M}_{\boldsymbol{d}}$. As shown in Fig. 1(c), the gyro moment matrix and mass matrix of the disc element at the mth node position are is follows:

$$
\begin{aligned}
\boldsymbol{D}_{d}=\omega\left[\begin{array}{cccc}
0 & 0 & 0 & 0 \\
0 & 0 & 0 & 0 \\
0 & 0 & 0 & J_{p} \\
0 & 0 & -J_{p} & 0
\end{array}\right] \\
\mathbf{M}_{d}=\operatorname{diag}\left[m_{d}, m_{d}, J_{d}, J_{d}\right] \quad m=6,7,8,9,20,22,24
\end{aligned}
$$

In the above formula, $\boldsymbol{m}_{\boldsymbol{d}}, \boldsymbol{J}_{\boldsymbol{d}}$ and $\boldsymbol{J}_{\boldsymbol{p}}$ are the mass, diameter moment of inertia and polar moment of inertia of the disc respectively.It can be calculated according to the following formula according to the disc material density and geometry (inner radius $r$, outer radius $R$ and thickness $h$ of the disc):

$$
m_{d}=\pi \rho(R-r)^{2} h \quad J_{d}=\frac{\rho \pi(R-r)^{4} h}{4} \quad J_{p}=\frac{\rho \pi(R-r)^{4} h}{2}
$$



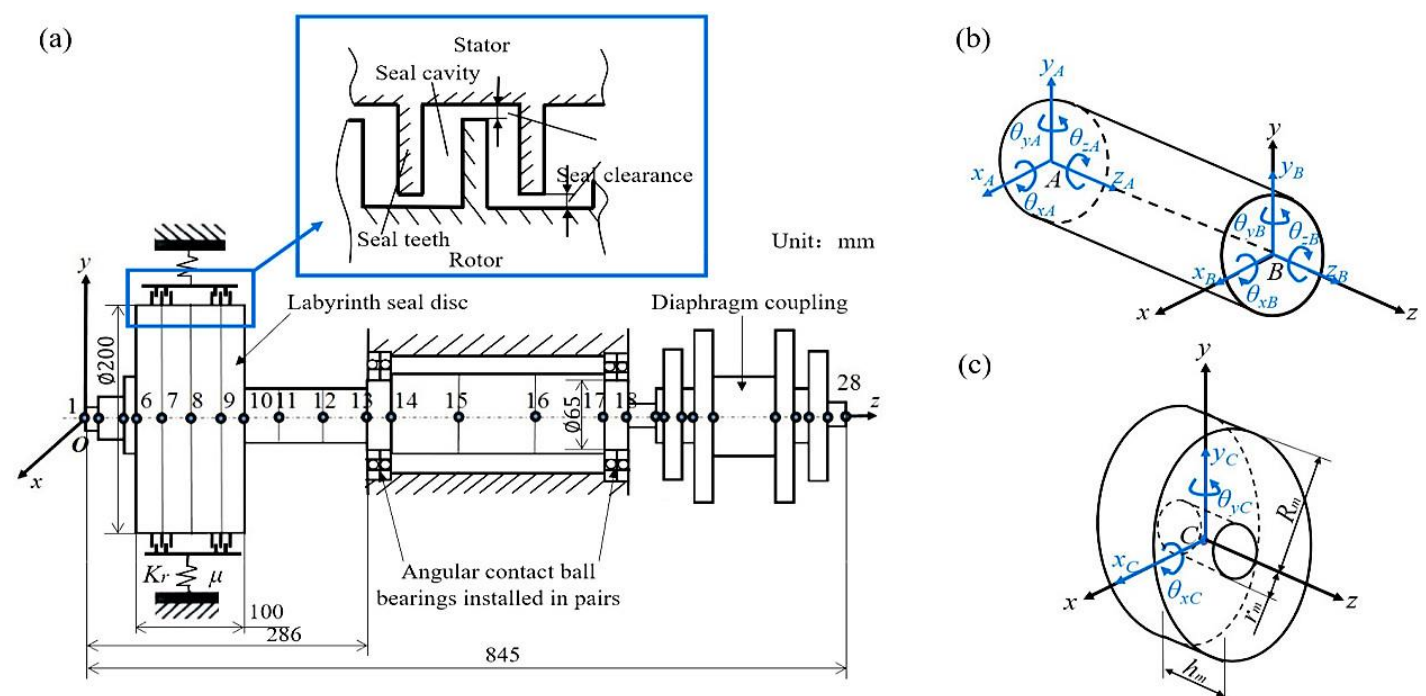

(c)

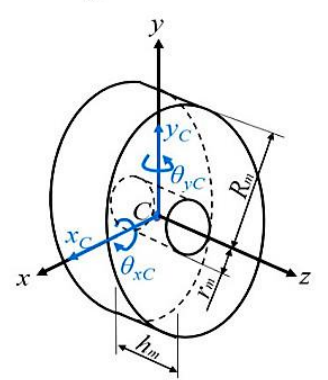

Fig. 1. (a) Model of labyrinth seal- rotor system; (b) FEM schematics of a shaft element; (c) FEM schematics of a rigid disk

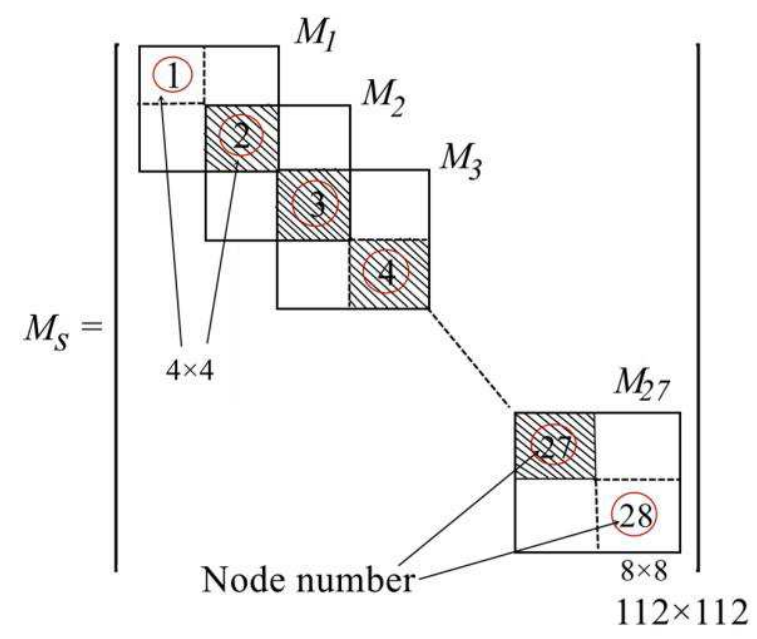

Fig. 2. Assembly of matrix.

\subsection{The establishment of the finite element model of the system crack element}

In the research, it is assumed that the moment of inertia of the crack section to the fixed coordinate axis of the system does not change with the change of the rotation angle. When the crack occurs, the moment of inertia of the crack to the two sections does not change, but it changes the center of mass of the section. Fig. 3 is a cross-sectional view of the crack unit, $o-x y$ is the fixed coordinate system of the rotating shaft, where $o$ is the center of the rotating shaft when there is no crack, $c$ is the center of the rotating shaft after the crack occurs, and $e$ is the position change of the center after the crack occurs, $\alpha$ Is the cracking angle of the crack, $h$ is the depth of the crack, and $R$ is the radius of the shaft. 


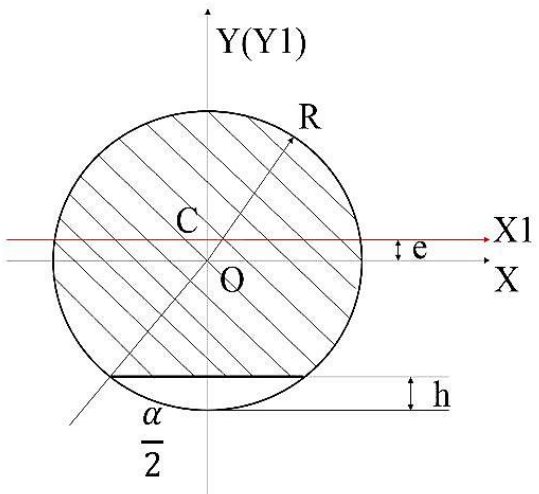

Fig. 3. Section of crack element.

The moment of inertia of the crack on the $o x$, oy coordinate axes is:

$$
\begin{aligned}
& I_{x}^{0}=\frac{\pi R^{4}}{8}-\frac{R^{4}}{4}(1-\mu) \quad\left(2 \mu^{2}-4 \mu+1\right) \gamma+\operatorname{si~}^{-1}(1-\mu) \\
& I_{y}^{0}=\frac{R^{4}}{12}(1-\mu) \quad\left(2 \mu^{2}-4 \mu-3\right) \gamma+3 \operatorname{si~}^{-1}(\gamma)
\end{aligned}
$$

The remaining area of the crack section is $A_{c e}$, the centroid moment $e$, and the crack angle $\alpha$ are:

$$
\begin{aligned}
& A_{c e}=R^{2}\left(\pi-\cos ^{-1}(1-\mu)+(1-\mu) \gamma\right)-\pi r^{2} \\
& e=\gamma^{3} \frac{2 R^{3}}{3 A_{c e}} \\
& \alpha=2 \operatorname{arcos}(1-\mu)
\end{aligned}
$$

In the above formula: crack depth ratio $\mu=h / R, \gamma$ is the dimensionless crack depth of the crack can be expressed as: $\gamma=\sqrt{\mu(2-\mu)}$.For the crack section, the moment of inertia of the remaining section except for the crack to the new coordinate axes $c x, c y$ is:

$$
\begin{aligned}
& I_{\bar{x}}^{c e}=I-\left(I_{x}^{0}+A_{c e} e^{2}\right) \\
& I_{\bar{y}}^{c e}=I-I_{y}^{0} \\
& I=\pi\left(R^{4} / 4\right)
\end{aligned}
$$

think $I_{1}=I_{x}^{0}+A_{c e} e^{2}, I_{2}=I_{y}^{0}$ The decrease in the moment of inertia can be expressed as:

$$
\begin{aligned}
& I_{\bar{x}}^{c e}=I-I_{1} \\
& I_{\bar{y}}^{c e}=I-I_{2}
\end{aligned}
$$

According to the finite element theory of rotor dynamics, it is believed that the crack occurs in the $l w$-th element. The matrix $\boldsymbol{K}^{l \boldsymbol{w}}{ }_{c}$ of the stiffness reduction of the crack element in the entire system is: 


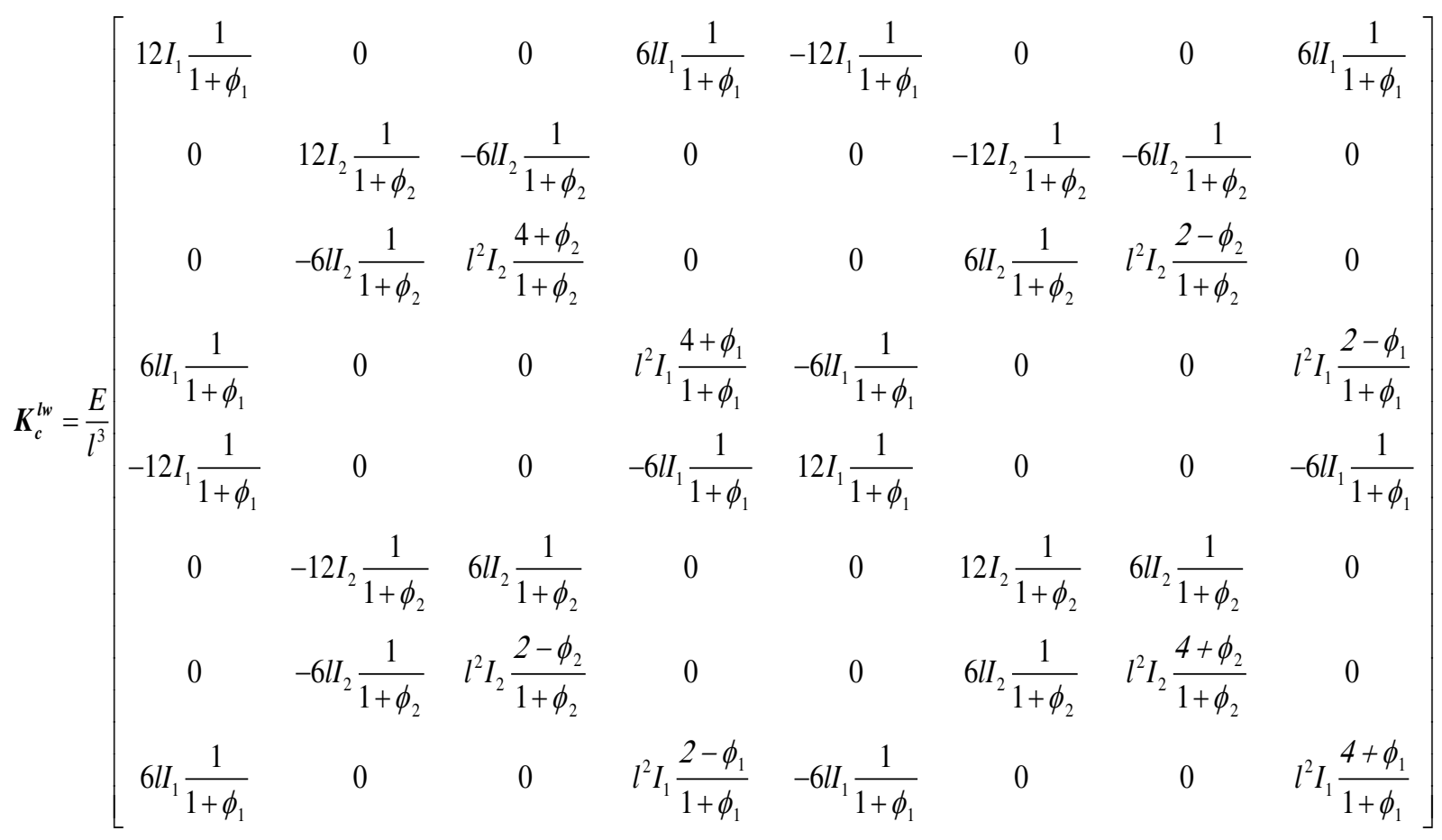

The stiffness model of the double crack mainly adopts the cosine stiffness model, and its formula is:

$$
\boldsymbol{K}=\boldsymbol{K}_{1}-f(t) \boldsymbol{K}_{c 1}^{l w}-f(t) \boldsymbol{K}_{c 2}^{l w}
$$

In the formula, $\boldsymbol{K}_{1}$ is the total stiffness of the system without cracks, $\boldsymbol{K}_{c 1}^{l w} 、 \boldsymbol{K}_{c 2}^{l w}$ is the stiffness reduction of the first crack and the second crack, and $f(t)$ is the switching function. The expression is:

$$
f(t)=\frac{1}{2}(1+\cos (\omega t+\beta))
$$

In the formula, $\beta$ is the phase difference angle of the double crack.

\subsection{Labyrinth seal-Muszynska sealing force model}

This paper selects a more accurate and practical sealing force model—Muszynska sealing force model[27]. This model introduces the average flow velocity ratio $\tau$ used to represent the fluid movement in the sealing gap, which expresses the sealing force vs The effect of inertia, damping, and stiffness produced by the rotor movement Fig. 4 is a radial cross-sectional view of the sealed cavity, where the circumferential angular velocity of the fluid close to the rotor is $\omega$, and it is 0 at the point close to the stator. The average flow rate ratio in the sealed cavity can be expressed as $\tau \omega$, and the sealing force can be expressed as:

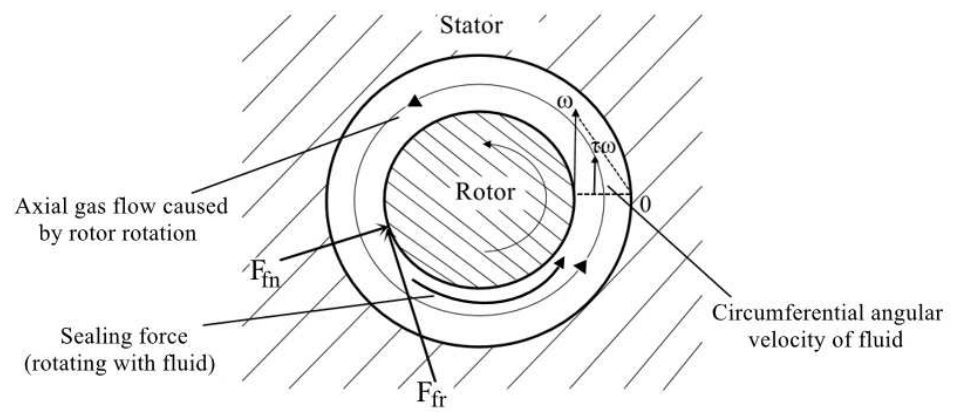

Fig. 4. Schematic diagram of Muszynska sealing force model. 


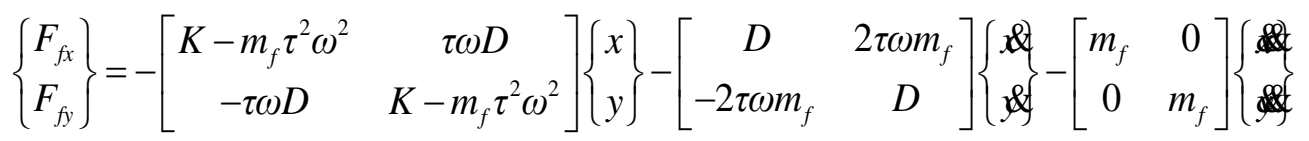

In the formula, $K 、 D 、 m_{f}$ are the stiffness, damping, and mass of the disturbance movement of the fluid to the rotor, respectively, and $\omega$ is the angular velocity of the rotor. $K 、 D 、 \tau$ are all nonlinear functions related to the disturbance displacement $x$ and $y$, which can be expressed in the following form:

$$
\begin{array}{ll}
K=K_{0}\left(1-e^{2}\right)^{-n} & \\
D=D_{0}\left(1-e^{2}\right)^{-n} & n=0.5: 3 \\
\tau=\tau_{0}(1-e)^{b} & 0<b<1, \tau_{0}<0.5 \\
e=\sqrt{x^{2}+y^{2}} / c &
\end{array}
$$

In the formula, $e$ is the relative eccentricity of the rotor, $c$ is the sealing gap (the distance between the rotor and the sealing teeth), $n 、 b 、 \tau_{0}$ are the specific sealing structure parameters.

$$
K_{0}=\mu_{0} \mu_{3} \quad D_{0}=\mu_{1} \mu_{3} T \quad m_{f}=\mu_{2} \mu_{3} T^{2}
$$

Among them $\mu_{0} 、 \mu_{1} 、 \mu_{2} 、 \mu_{3}$ and $T$ are respectively expressed as:

$$
\begin{aligned}
& \mu_{0}=\frac{2 \sigma^{2}}{1+z+2 \sigma} E_{f}\left(1+m_{0}\right) \\
& \mu_{1}=\frac{2 \sigma^{2}}{1+z+2 \sigma}\left[\frac{E_{f}}{\sigma}+\frac{B}{2}\left(E_{f}+\frac{1}{6}\right)\right] \\
& \mu_{2}=\frac{\sigma\left(E_{f}+\frac{1}{6}\right)}{1+z+2 \sigma} \\
& \mu_{3}=\frac{\pi R_{m} \Delta P}{\lambda} \\
& T=\frac{l}{v_{a}}
\end{aligned}
$$

Among them $\sigma, \lambda 、 E_{f}$ and $B$ are respectively expressed as:

$$
\begin{aligned}
& \sigma=\frac{\lambda l}{\mathrm{c}} \\
& \lambda=n_{0} R_{a} m_{0}\left[1+\left(\frac{R_{v}}{R_{a}}\right)^{2}\right]^{\frac{1+m_{0}}{2}} \\
& E_{f}=\frac{1+z}{1+z+2 \sigma} \\
& B=2-\frac{\left(\frac{R_{v}}{R_{a}}\right)^{2}-m_{0}}{\left(\frac{R_{v}}{R_{a}}\right)^{2}+1}
\end{aligned}
$$




$$
R_{v}=\frac{R_{m} c \omega}{\gamma} \quad R_{a}=\frac{2 v_{a} c}{\gamma}
$$

$z$ represents the inlet loss coefficient, $\gamma$ represents the hydrodynamic viscosity coefficient, $\sigma$ represents the friction loss gradient coefficient, $R_{V}$ represents the circumferential flow Reynolds number, $R_{a}$ represents the axial flow Reynolds number, $V_{a}[29]$ represents the axial flow velocity of the airflow, and $\Delta P$ represents the seal pressure difference. $R_{m}$ represents the sealing radius, c represents the sealing gap. Other parameters can be seen in Table 1. 2.4 Dynamic equations of the seal-rotor system

The mass, stiffness, and gyro matrix of each unit of the system obtained are merged into the mass, gyro matrix, and stiffness of the entire system, namely $\boldsymbol{M} 、 \boldsymbol{D} 、 \boldsymbol{K}_{\boldsymbol{l}}$. The damping of the system adopts proportional damping, that is, damping $\boldsymbol{C}$ is a linear combination of $\boldsymbol{M}$ and $\boldsymbol{K}$, and its expression is:

$$
\begin{aligned}
& \boldsymbol{C}=\alpha \boldsymbol{M}+\beta \boldsymbol{K}_{\boldsymbol{1}} \\
& \alpha=4 \pi f_{1} f_{2}\left(\xi_{2} f_{1}-\xi_{1} f_{2}\right) /\left(f_{1}^{2}-f_{2}^{2}\right) \\
& \beta=\left(\xi_{2} f_{2}-\xi_{1} f_{1}\right) / \pi\left(f_{2}^{2}-f_{1}^{2}\right)
\end{aligned}
$$

$\xi_{1}$ and $\xi_{2}[28]$ are damping coefficients, representing the damping ratio of the first and second modes of the system, $f_{1}$ and $f_{2}$ are the first and second frequencies of the system, so the dynamic equation of this system is:

$\boldsymbol{M}(\boldsymbol{D}+\boldsymbol{C}) \&+\left(\boldsymbol{K}_{\boldsymbol{I}}-F(t) \boldsymbol{K}_{c}^{l w}\right) x=\boldsymbol{Q}$

Among them, $\boldsymbol{Q}=\boldsymbol{F}_{\boldsymbol{p}}+\boldsymbol{F}_{\boldsymbol{f}}+\boldsymbol{G}$ is the sum of eccentric force, gravity, and sealing force. The expression of eccentric force $\boldsymbol{F}_{\boldsymbol{p}}$ is:

$$
\begin{aligned}
& \boldsymbol{F}_{\boldsymbol{P}}=m e \omega^{2} \\
& \boldsymbol{F}_{\boldsymbol{P} \boldsymbol{x}}=m e \omega^{2} \cos (\omega t) \\
& \boldsymbol{F}_{\boldsymbol{P y}}=m e \omega^{2} \sin (\omega t)
\end{aligned}
$$

Table 1 Parameters of the seal-bearing- rotor system.

\begin{tabular}{lcc}
\hline & parameter & value \\
\hline & $E(\mathrm{~Pa})$ & $2.1 \times 10^{11}$ \\
& $\rho\left(\mathrm{Kg} / \mathrm{m}^{3}\right)$ & 7850 \\
Rotor system model parameters & $v$ & 0.3 \\
& $\xi_{1}, \xi_{2}$ & $0.02,0.04$ \\
& $m e(\mathrm{~g} \cdot \mathrm{mm})$ & 600 \\
& $f_{l}, f_{2}(\mathrm{~Hz})$ & $62.809,123.55$ \\
\hline \multirow{3}{*}{ Crack parameters } & $\mu=h / R$ & $1 / 6 、 1 / 2$ \\
& $h$ & $5 、 15$ \\
& $R$ & 30 \\
\hline & $c(\mathrm{~mm})$ & 0.1 \\
& $l_{m}(\mathrm{~mm})$ & 15 \\
$R_{m}(\mathrm{~mm})$ & 100 \\
$\Delta P(\mathrm{MPa})$ & 0.3
\end{tabular}




$$
\begin{gathered}
\gamma(\mathrm{Pa} \cdot \mathrm{s}) \\
z
\end{gathered}
$$

Experience coefficient $m_{0}[28]$

Experience coefficient $n_{0}[\mathbf{2 8}]$

Experience coefficient $\tau_{0}[28]$

Experience coefficient $n_{f}[30]$

Experience coefficient $b[29]$
$1.79 \times 10^{-5}$

0.1

$-0.25$

0.079

0.2

2.5

0.45

\section{The effect of a single crack on the seal-rotor system}

\subsection{The influence of the degree of crack damage on the rotor system}

Fig. 5 shows the response diagram of the system at the No. 10 shaft section at the damage position when the speed of the system increases with different degrees of crack damage. Fig. 5(a1)-(a2) are the bifurcation diagram and three-dimensional waterfall diagram of the system when the crack depth ratio is $1 / 6$. In the bifurcation diagram, it can be seen that the system is making a cycle-one motion. In the three-dimensional waterfall diagram, it can be seen that the system appears The weak double frequency component is removed, at this time the system frequency component is only $1 X, 2 X$; Fig. 5(b1)-(b2) are the bifurcation diagram and three-dimensional waterfall diagram of the system when the crack depth ratio is $1 / 2$. In the bifurcation diagram, it can be seen that the system has more obvious fluctuations in the first-order critical speed. And there is a slight fluctuation at the lower speed. $1 X, 2 X, 3 X$, $4 X$ high frequency appear in the three-dimensional waterfall chart and $2 X$ at speeds equal to $2550 \mathrm{rev} / \mathrm{min}$ and $1275 \mathrm{rev} / \mathrm{min}$. There are two peaks and a peek at $850 \mathrm{r} / \mathrm{min}$ in $3 X$.

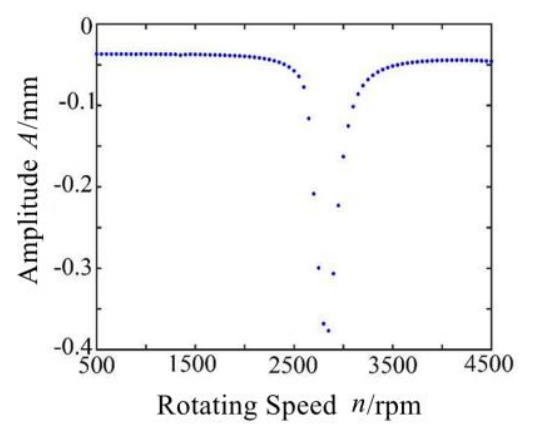

(a1)Bifurcation diagram

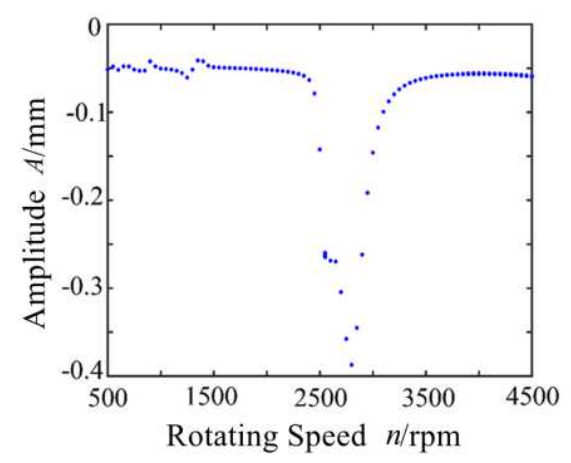

(b1)Bifurcation diagram

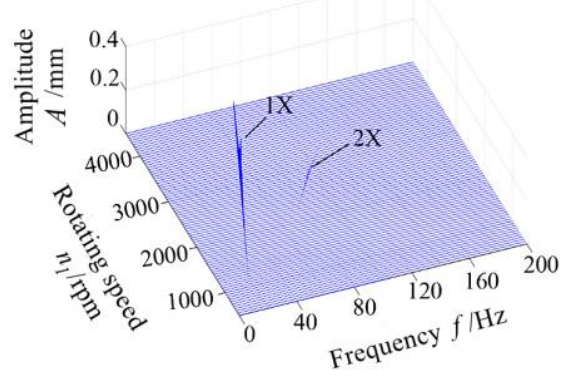

(a2)3D waterfall chart

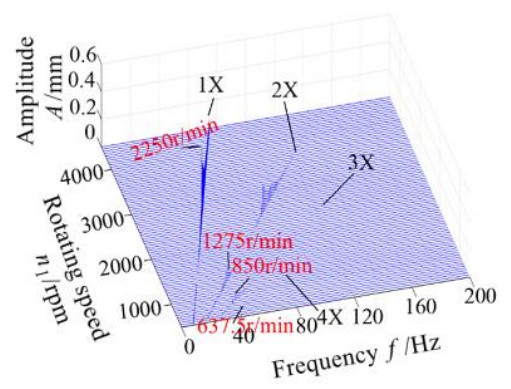

(b2)3D waterfall chart

Fig.5. Bifurcation diagram and 3D waterfall chart of different crack damage(a) $\mu=1 / 6(b) \mu=1 / 2$ 
This section mainly analyzes the impact of crack damage on the system when the airflow force appears in the seal-rotor system, and the system speed increases to $10000 \mathrm{rev} / \mathrm{min}$. Fig. 6 is the response diagram of the system when there is no crack. In the figure, it can be seen that when the system speed reaches $6300 \mathrm{r} / \mathrm{min}$, the system will lose stability and enter the pseudo-periodical state, and there will be a period-doubling movement in the range of 7600rev/min-7900rev/min periodic window phenomenon.

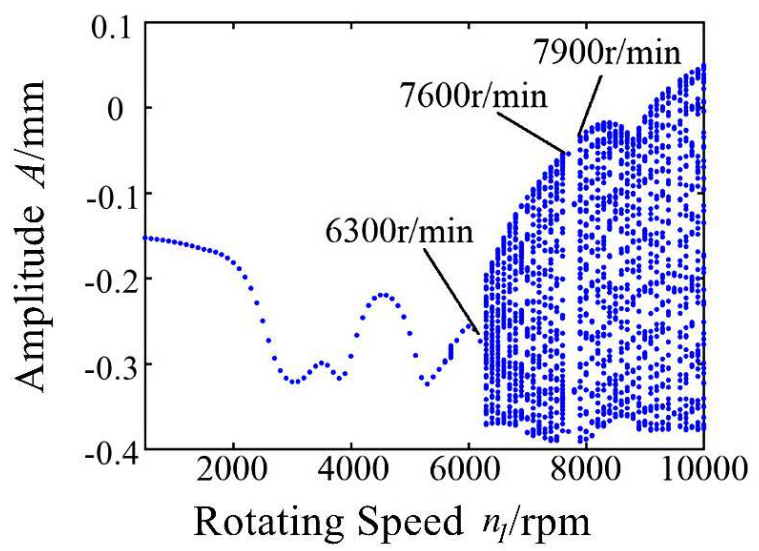

Fig. 6. Bifurcation diagram of the system without crack.

When there is airflow, the air pressure at the sealing outlet is $0.1 \mathrm{Mpa}$ Fig. 7(a) is a bifurcation diagram of different crack damage degrees bifurcation diagrams are respectively when the crack depth ratio $\mu$ is $1 / 6$ and 1/2. It can be seen that when cracks appear, the system's destabilizing speed decreases significantly from 6300r/min (no crack damage) to 6100rev/min (crack failure occurs). As the crack deepens, the destabilizing speed also gradually decreases. When the depth of the crack increases to a certain extent, the instability speed increases slightly, and the period window of the system continues to advance as the damage degree of the system continues to increase. Fig. 7(b) is the three-dimensional waterfall diagram under different crack damage degrees, respectively is the three-dimensional waterfall diagram of the system when $\mu$ is $1 / 6,1 / 2$. It can be seen that the three-dimensional waterfall diagram mainly has $1 X, 2 X$, and $3 X$ frequency multiplication Composition, airflow excitation frequency ff and some combination frequencies such as $1 X+f f, 1 X+2 f f, 1 X-f f, 2 X+f f, 2 X+2 f f$, $2 X-f f$, etc.

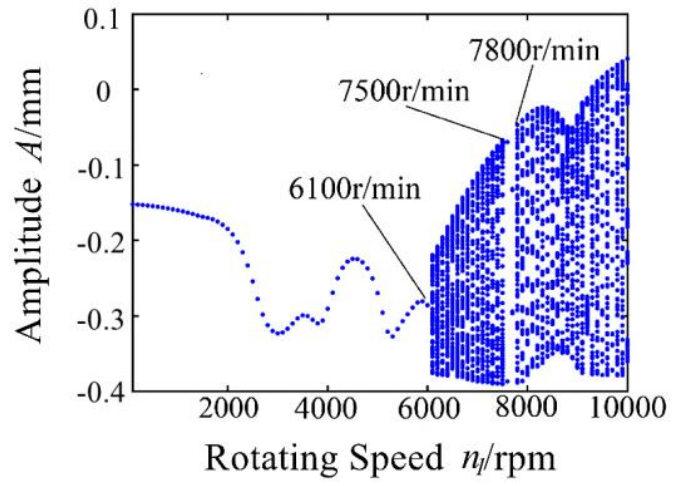

(a1)Bifurcation diagram of $\mu=1 / 6$

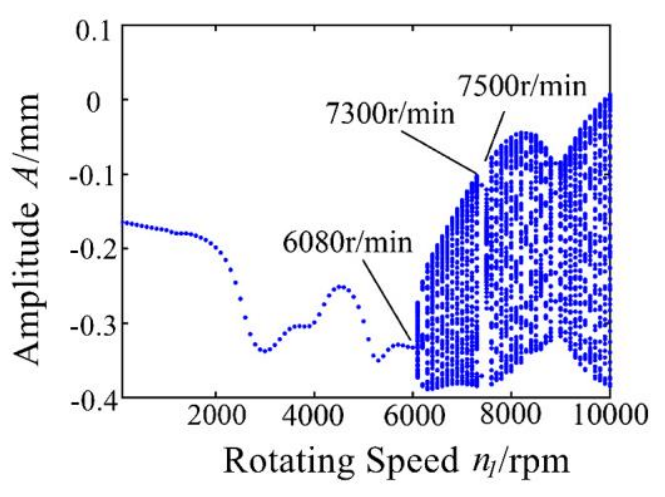

(a2)Bifurcation diagram of $\mu=1 / 2$ 


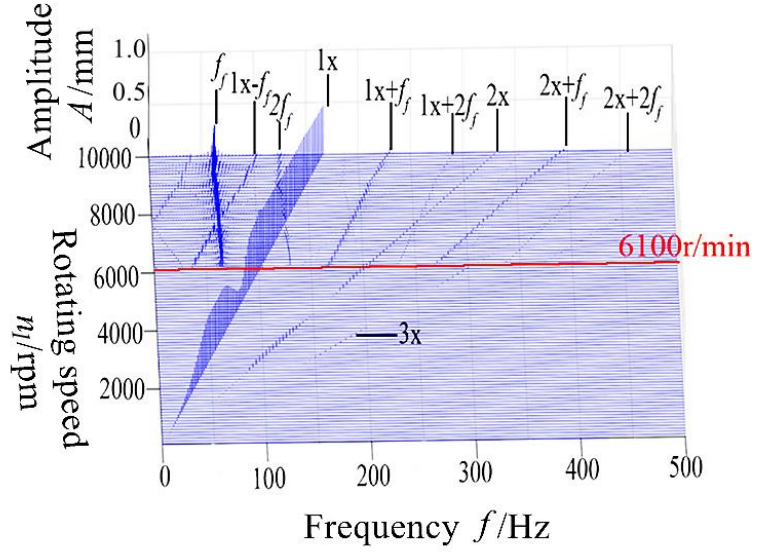

(b1)3D waterfall chart of $\mu=1 / 6$

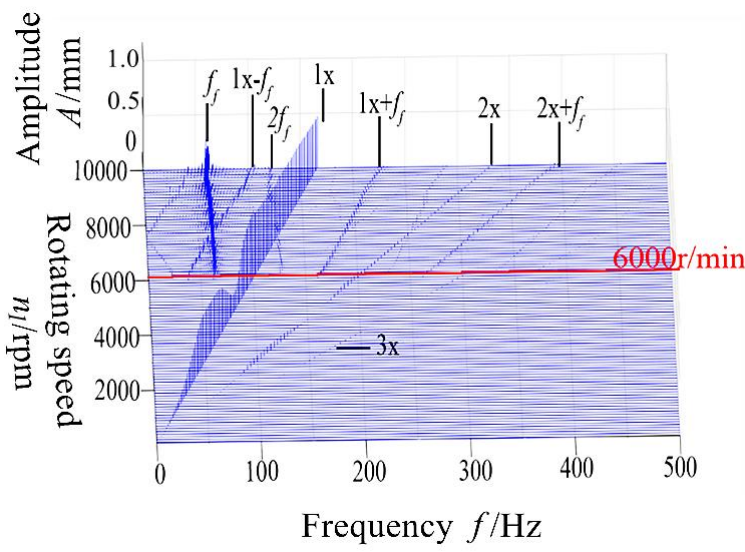

(b2)3D waterfall chart of $\mu=1 / 2$

Fig.7. Response graph of different crack damage degree (a) Bifurcation diagram (b) 3D waterfall chart

Fig. 8 is the amplitude curve of the seal-rotor system with crack damage failure during the speed-up process when there is no crack damage failure. It can be seen from Fig. 7 that when the rotational speed is greater than $6000 \mathrm{r} / \mathrm{min}$, airflow excitation will occur. Among them, the amplitude curve of cracks has a crack depth ratio of $\mu=1 / 2$. It can be seen that the amplitude of a rotor system with cracks is significantly higher than that of a rotor system without cracks when there is no airflow excitation. However, the amplitude of the rotor system with cracked failure after the airflow excitation of the system is not much different from that of the non-cracked rotor system. This is because the airflow force has a certain inhibitory effect on the crack failure, and the system is in a high-speed area. The dynamic behaviour is dominated by airflow excitation.

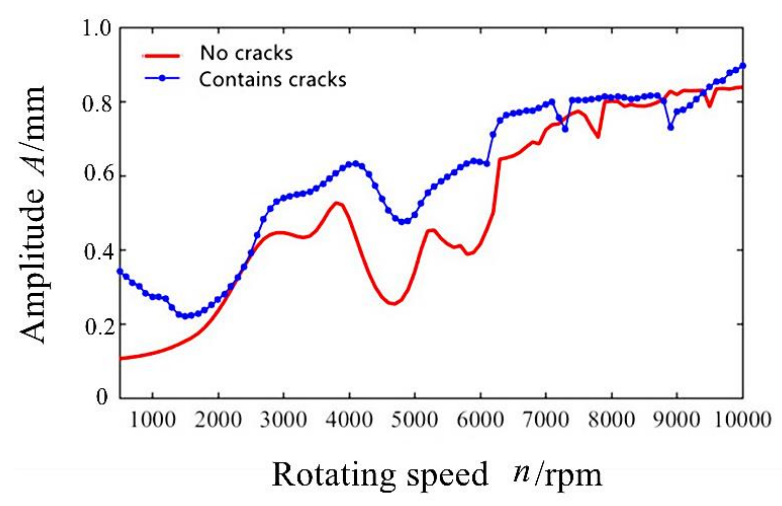

Fig. 8. Amplitude curve of system with or without crack.

\subsection{The effect of crack damage on the system under steady-speed conditions}

Fig. 9 is a comparison diagram of the axis trajectory and time-domain waveform of the $1 / n(n=1,2)$ order critical speed when the $\mu=1 / 2$ system is with or without airflow excitation force. When the system has no air force, the response is red, and when there is the air force, the axis track is blue. It can be seen that the amplitude when there is air force is significantly lower than that when there is no air force. When the system speed is at other values, the movement range of the axis trajectory is smaller when there is airflow force than when there is no airflow force. The amplitude of vibration decreases and the waveform is more stable when there is airflow force in the domain waveform diagram. It also shows that the airflow excitation force will weaken the 
high-frequency vibration caused by cracks in the subcritical speed region and reduce the system amplitude.

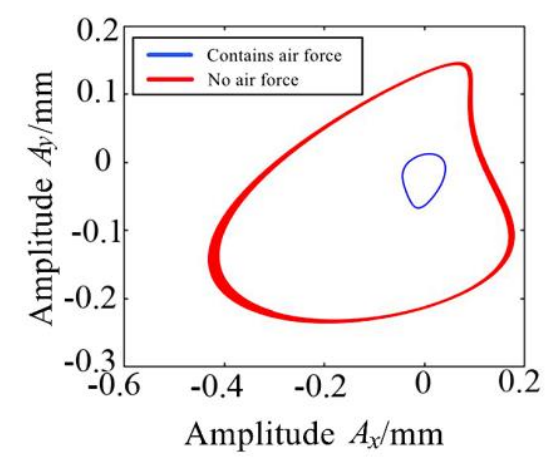

(a1)Axis trajectory diagram

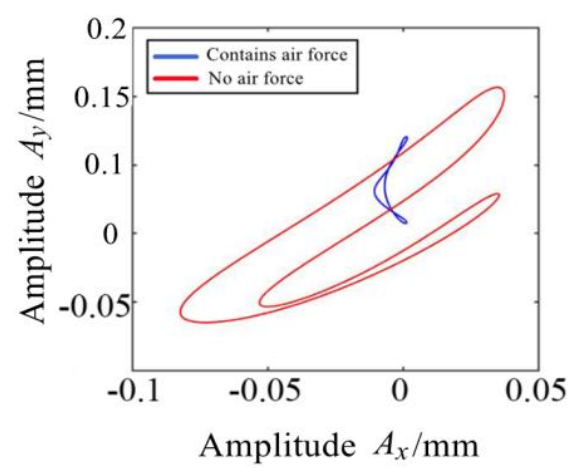

(b1)Axis trajectory diagram

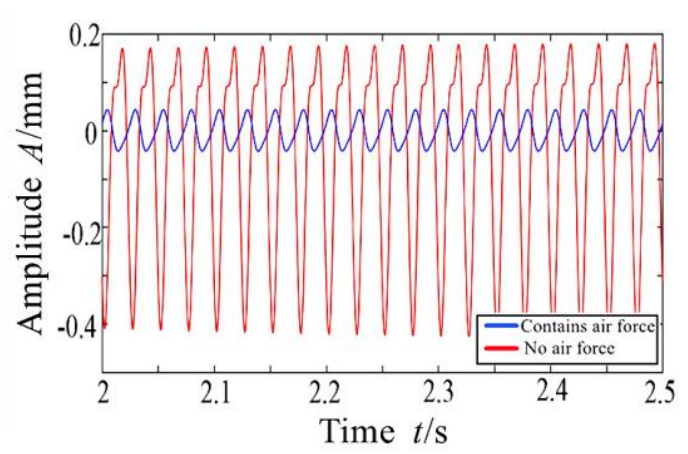

(a2)Time domain waveform

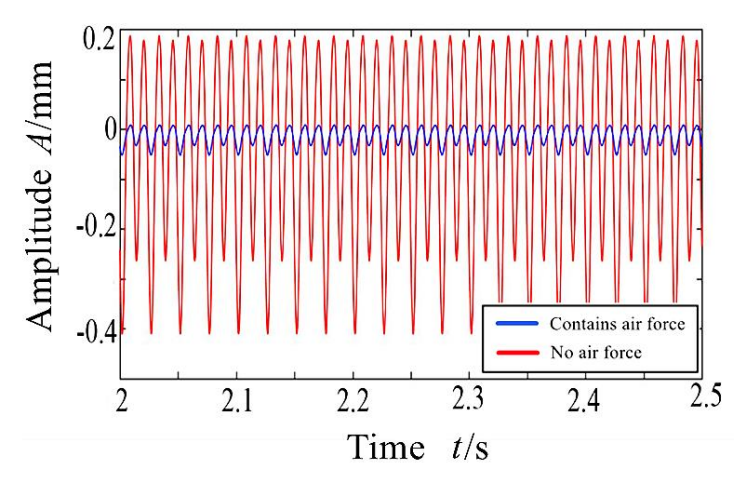

(b2)Time domain waveform

Fig. 9. Rotor system with or without air force response diagram(a)2550 rev/min(b)1275rev/min

\subsection{Test verification}

In order to verify the correctness of the theoretical modelling and numerical simulation results, the built-up seal-rotor test bench is shown in Fig.10, and the schematic diagram of the hardware structure can be seen in Fig. 10(a). The test bench is composed of a drive system, a supporting system, a test piece system, a lubrication system, a pneumatic system, and an electric control system. The test bench uses a motor with a power of up to $15 \mathrm{KW}$ and a maximum speed of $6000 \mathrm{rev} / \mathrm{min}$ and uses a frequency converter to control the speed of the motor. Fig. 10(b) shows a partial cross-sectional view of the test piece. The airflow flows in from the air inlet in the middle and flows out from the front and back ends. There is a staggering labyrinth seal structure at the front and back. Each seal structure contains 3 sealing teeth. The sealing length is $15 \mathrm{~mm}$.

The test system composition and installation position of the seal-rotor test bench is shown in Fig. 10(c). A pair of eddy current sensors, a group of photoelectric sensors and acceleration sensors are arranged at the corresponding positions of the test bench. After the amplifier conditions the signal through the signal cable, the signal is sent to the dynamic data acquisition instrument, and the steps of analogue signal anti-mixing filtering and $\mathrm{A} / \mathrm{D}$ conversion are realized in the data acquisition instrument. It is finally converted into a digital signal that the upper-level analysis software can process. Finally, the digital signal is uploaded to the analysis software of the computer to realize 
various analysis functions.

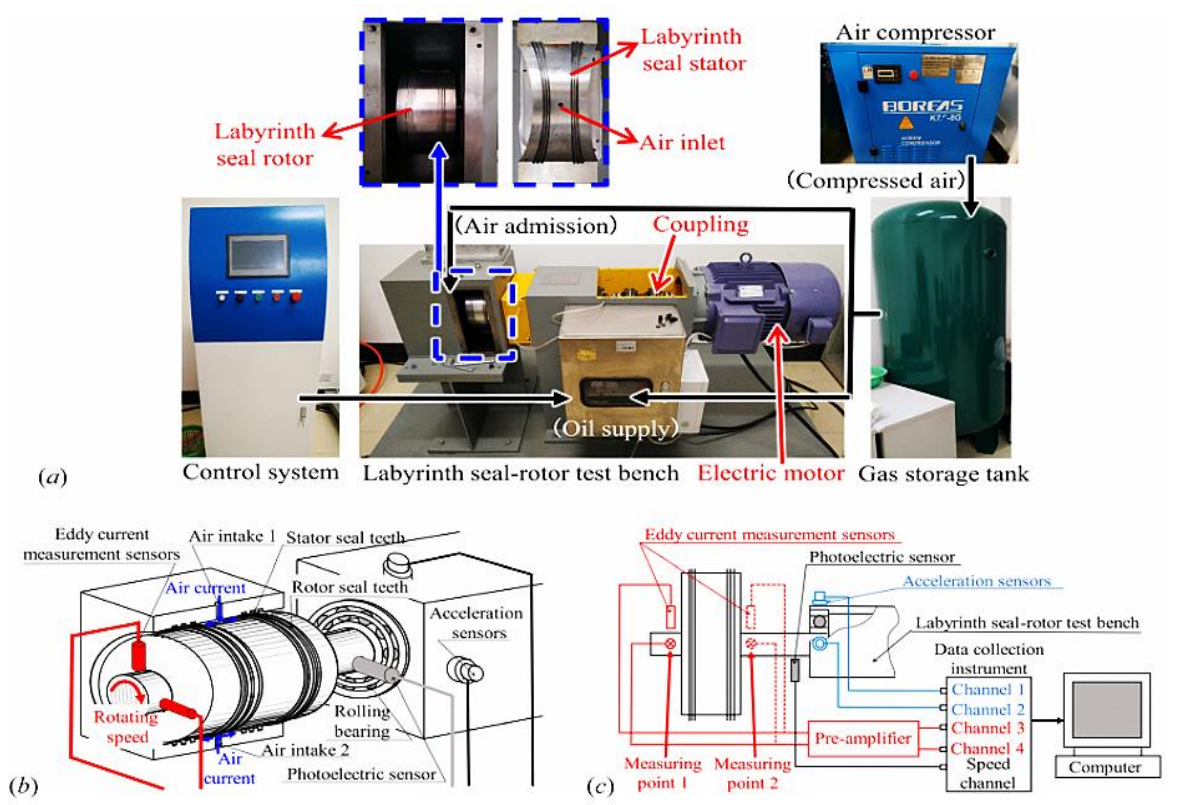

Fig. 10. Seal-rotor test rig: (a) schematic of hardware; (b) sectional view of the test specimen; (c) testing system.

In the experimental test of the labyrinth seal-rotor system, the impact of the system's crack damage failure in the subcritical speed region on the vibration characteristics of the system is mainly analyzed and compared with the crack failure characteristics of the rotor system in the subcritical speed region during simulation calculations. The degree and location of the crack damage of the experimental study of the sealing shaft are the same as the simulation calculation $\mu=0.5$, and the damage location is the No. 10 shaft section.Fig. 11 is a comparison diagram of system vibration response simulation and experimental test in the subcritical speed region. Fig. $11 \mathrm{~s}(\mathrm{a} 1)-(\mathrm{a} 2)$ and(b1)-(b2) are the first-order critical speed, and Fig. 11s(c1)-(c2)and(d1)-(c2) are 1/2 times the critical speed. The fault characteristic of the crack obtained in the simulation calculation is that the system has a high-frequency doubler and the frequency doubler phenomenon appears at the $1 / 2$ critical speed in the subcritical speed region (system super-harmonic vibration). The axis trajectory is also the same as the axis during simulation. The trajectory is similar.

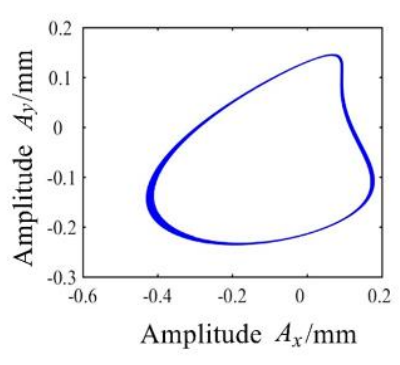

(a1)Axis trajectory diagram

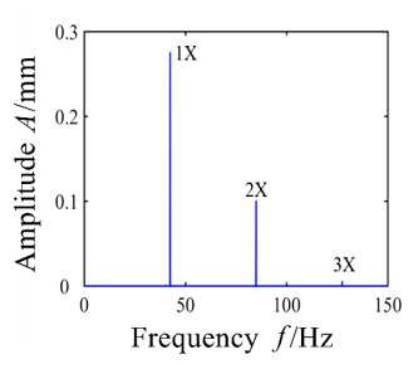

(a2)FFT spectrum

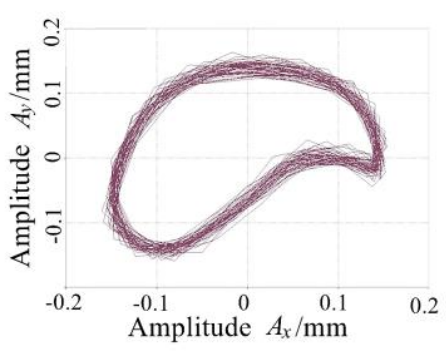

(b1)Axis trajectory diagram

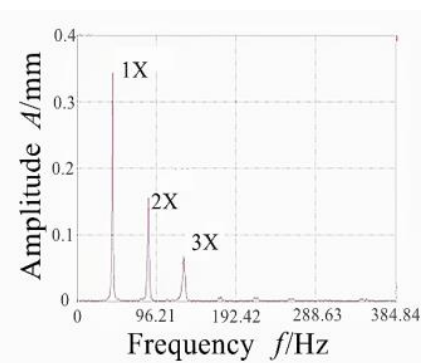

(b2)FFT spectrum 


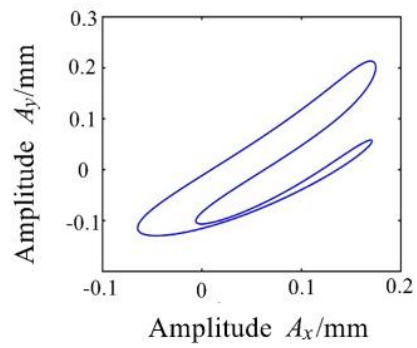

(c1)Axis trajectory diagram

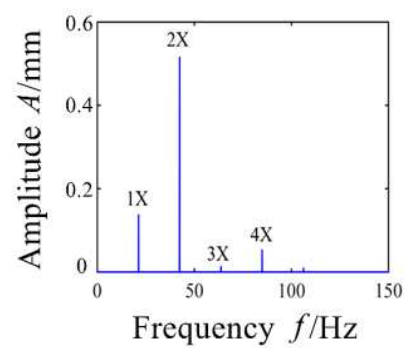

(c2)FFT spectrum

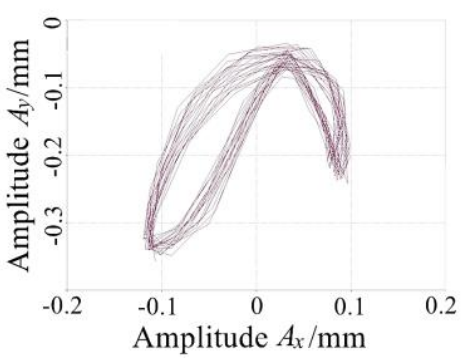

(d1)Axis trajectory diagram

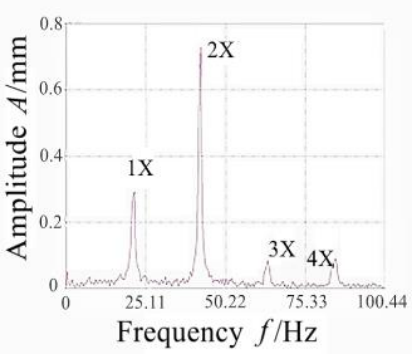

(d2)FFT spectrum

Fig. 11.Simulation and experimental response graphs at different speeds (a) 2550rev/min (b) 2620rev/min(experiment) (c)1275rev/min(d) 1300rev/min(experiment)

\section{The effect of cracks on different sealing parameters of the system}

\subsection{Effect of damage on changing seal pressure difference}

The relevant calculation parameters in this section are the same as those in the previous chapter. The air pressure difference between the sealed chamber and the outside can be achieved by changing the inlet pressure of the sealed inlet. It mainly analyzes the response characteristics of the system when the system's sealing pressure difference is $0.2 \mathrm{Mpa}$ and $0.3 \mathrm{Mpa}$ when the operating speed is increased from $100 \mathrm{rev} / \mathrm{min}$ to $10000 \mathrm{rev} / \mathrm{min}$.(see Fig. $12 \mathrm{~s}(\mathrm{a} 1)-(\mathrm{a} 2)$ and(b1)-(b2) is the bifurcation diagram and three-dimensional waterfall diagram of the system under different sealing pressure differences. In the bifurcation diagram, it can be seen that the system's instability speed is also increased with the increase of the sealing pressure difference. In the three-dimensional waterfall diagram of the system, it can be seen that there are high-frequency $2 X, 3 X$ caused by crack faults, and there are also the airflow excitation frequency ff that occurs after the system instability enters the pseudo-period state, and the combined frequency after the airflow excitation occurs. For example: $2 f f, 1 X-f f, 1 X+f f, 1 X+2 f f, 1 X-2 f f, 2 X+f f, 2 X+2 f f$ and other complex frequencies. However, the combined frequency components of the system instability in the three-dimensional waterfall diagram are continuously reduced with the continuous increase of the seal pressure difference.

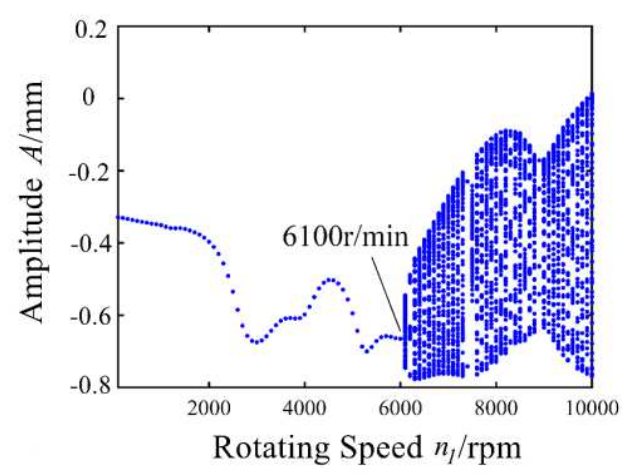

(a1)Bifurcation diagram

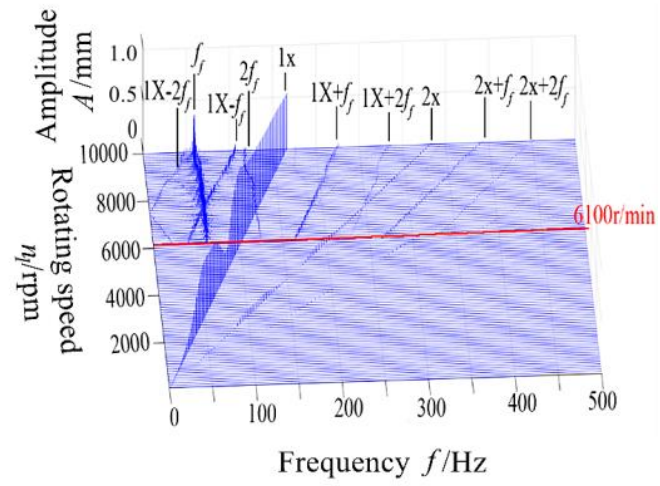

(a2)3D waterfall chart 


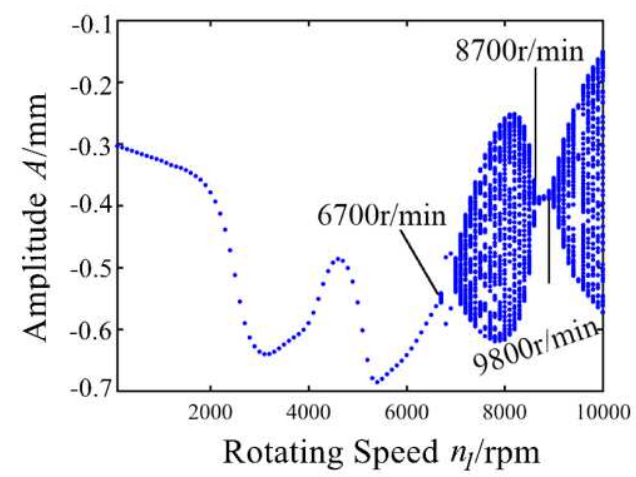

(b1)Bifurcation diagram

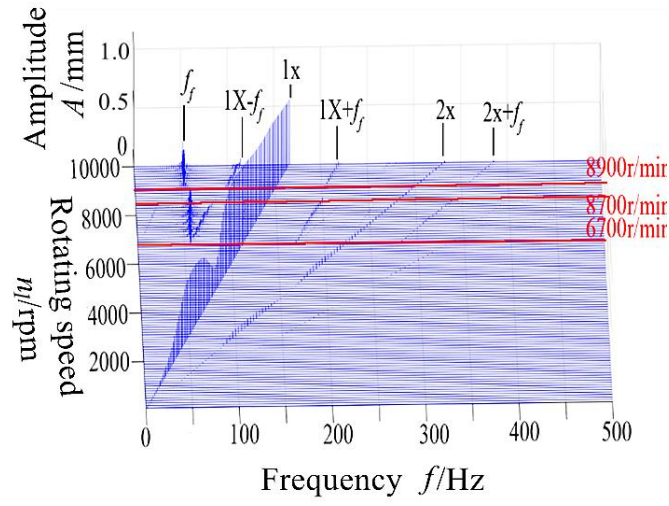

(b2)3D waterfall chart

Fig. 12. Bifurcation diagram and $3 \mathrm{D}$ waterfall diagram at different sealing pressure differences(a)0.2Mpa(b)0.3Mpa

\subsection{Response characteristics of variable seal pressure difference to steady speed of cracked system}

The system speed is stabilized at $10000 \mathrm{rev} / \mathrm{min}$, and the response of the system within the range of 0.1Mpa-1Mpa is analyzed. From Figure Fig. 13s(a)and(b), it can be seen that the system performs pseudo-periodic motion in the range of $0.1 \mathrm{Mpa}-0.38 \mathrm{Mpa}$. In the three-dimensional waterfall diagram, it can be seen that the frequencies in the range are: ff, $1 X, 2 X, 3 X, 2 f f, 1 X-f f, 1 X+f f, 1 X+2 f f, 1 X-2 f f$, etc., and as the seal pressure difference increases, the system airflow excitation frequency ff also decreases, from $70 \mathrm{~Hz}$ to $50 \mathrm{~Hz}$. When the seal pressure difference is after $0.38 \mathrm{Mpa}$, the system enters the cycle one movement and no airflow excitation occurs, and its frequency in the three-dimensional waterfall chart only has $1 X, 2 X$, and $3 X$ components. Fig. 13(c) is the amplitude curve of the system. It can be seen that the system amplitude was relatively stable before $0.18 \mathrm{Mpa}$, and then the system amplitude began to drop sharply and ended in a stable state at $0.38 \mathrm{Mpa}$.

(a)

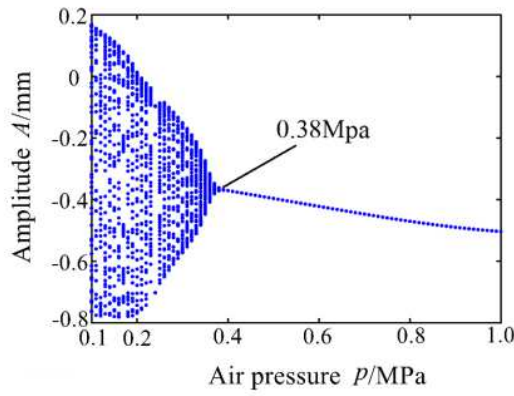

(b)

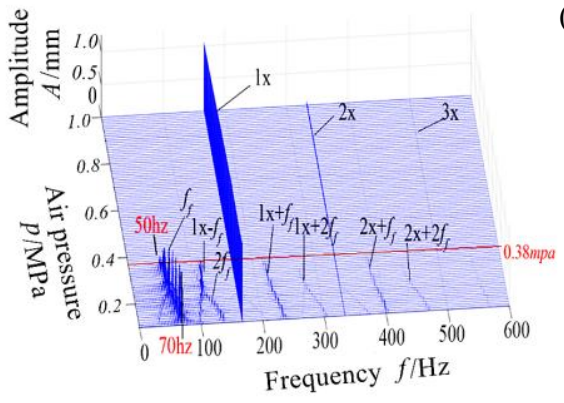

(c)

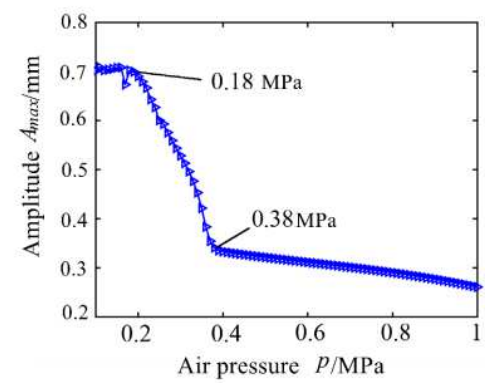

Fig. 13. The system response diagram with the change of seal pressure difference (a) Bifurcation diagram (b) 3D waterfall chart(c)Amplitude curve

\subsection{Response characteristics of variable seal length to steady speed of cracked system}

The operating speed of the system is stabilized at $10000 \mathrm{r} / \mathrm{min}$, and the change of the sealing length is controlled by increasing or decreasing the number of sealing teeth. The sealing length is used as a variable to analyze the response characteristics of the system at this time. In the bifurcation diagram Fig. 14(a), it can be seen that when the system is at a steady speed, the system performs a pseudo-periodical motion within the range of 
$10 \mathrm{~mm}$ to $22 \mathrm{~mm}$, and the system enters a stable cycle motion after the seal length increases to $22 \mathrm{~mm}$; In the three-dimensional waterfall chart of the method, it can be seen that the sudden change of the airflow excitation frequency $\mathrm{ff}$ of the system at the seal length of $12 \mathrm{~mm}$, when the length is $10 \mathrm{~mm}$, the excitation frequency is 134.2hz and gradually decreases with the increase of the seal length and is at $12 \mathrm{~mm}$. A sudden change to $70 \mathrm{hz}$ occurs. When the seal length is greater than $22 \mathrm{~mm}$, the airflow excitation frequency of the system disappears, and the system only has power frequency and doubling frequency components and enters a stable motion state. In the amplitude curve of the system Fig. 14(b), it can be seen that the amplitude of the seal length increases slightly before $14.5 \mathrm{~mm}$ and then begins to decrease, and the amplitude starts to remain stable when the system enters the cycle at $22 \mathrm{~mm}$. From the axis trajectory diagram of the Fig. 14(c) system, it can be seen that as the seal length increases, the axis trajectory changes from a complex nested large circular ring shape to a single circular ring.

(a)

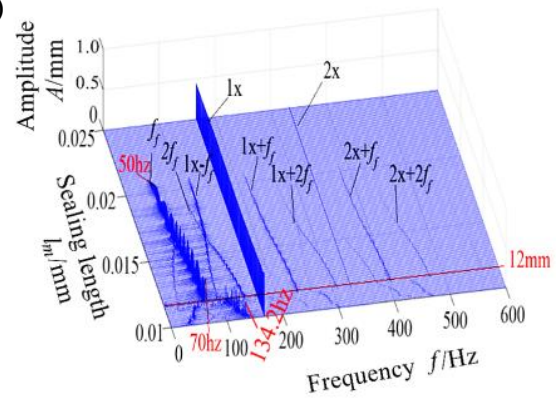

(b)

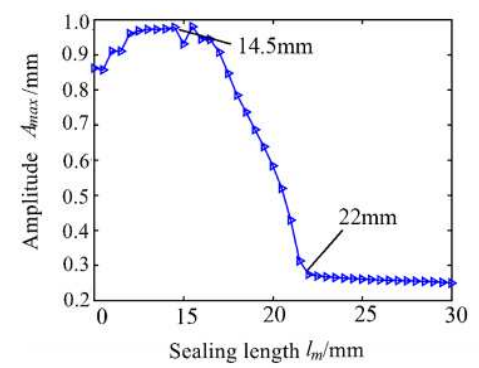

(c)

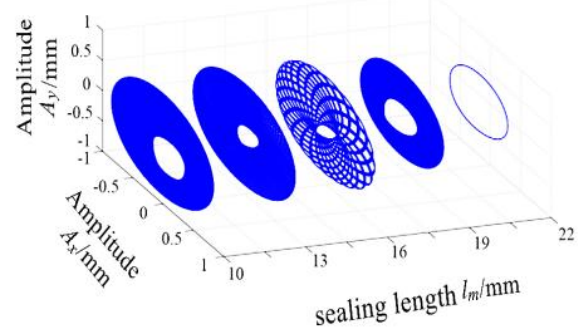

Fig. 14. System response as the seal length changes(a)3D waterfall chart(b)Amplitude curve(c)Axis track diagram

\subsection{The effect of damage changes on the sealing gap}

This section mainly analyzes the system's response characteristics when the sealing gap is $c=0.08 \mathrm{~mm}$ and $c=0.12 \mathrm{~mm}$. Figure 16 is a three-dimensional waterfall diagram of the system when the system speed is increased from 100r/min to 10000r/min under the two types of sealing gaps. It can be found from Figure Fig. 15 that before the system was unstable, there were only $1 \mathrm{X}, 2 \mathrm{X}$, and $3 \mathrm{X}$ multiplier components in the frequency of the system. After the system is unstable, the system starts to appear the airflow excitation frequency ff and some combined frequencies $1 X-f f$, $1 X-2 f f, 1 X+f f, 1 X+2 f f$, $2 f f-1 X, 2 X+f f$, etc., when the speed increases to $8800 \mathrm{r} / \mathrm{min}$, the airflow excitation vibration has a sudden change. When the sealing gap increases to $0.12 \mathrm{~mm}$ Fig. $15(\mathrm{~b})$, the combined frequency component is continuously decreasing. It reflects that the increase of the sealing gap increases the amount of gas leakage. The increase of the speed also enhances the fluidity of the gas, weakens the phenomenon of airflow excitation in the sealed cavity, and increases the sealing gap is conducive to the stability of the system. 
(a)

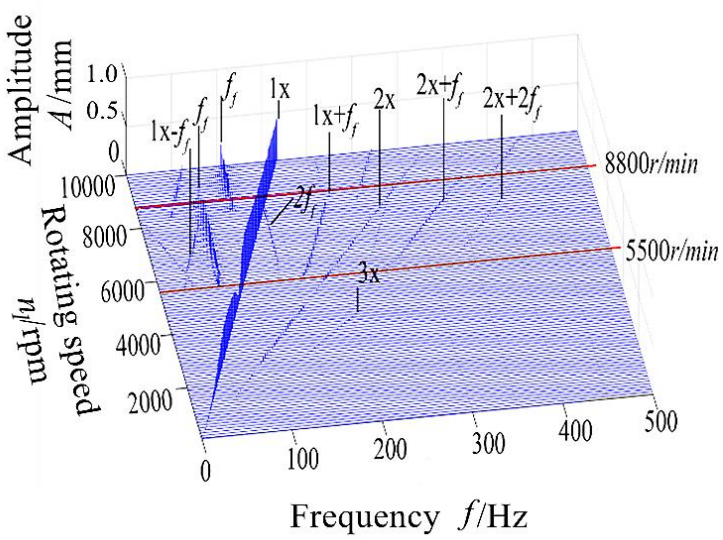

(b)

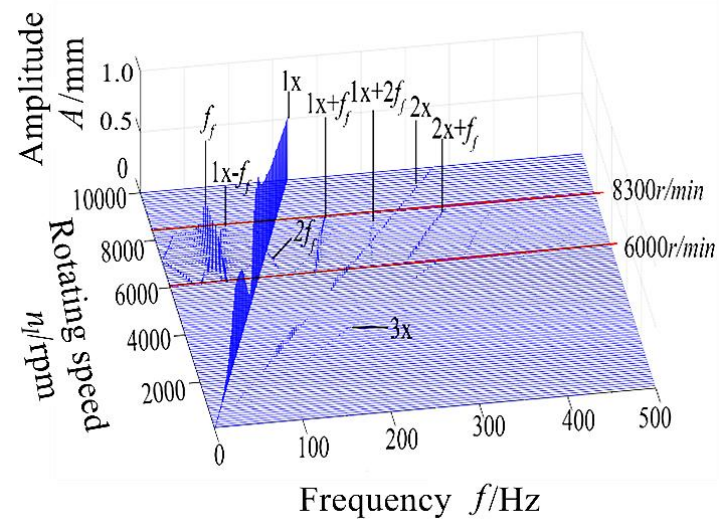

Fig. 15.3D waterfall chart of different gaps(a) $c=0.08 \mathrm{~mm}(\mathrm{~b}) c=0.12 \mathrm{~mm}$

\subsection{Response Characteristics of Variable Sealing Gap to Steady Speed of Cracked System}

Keep the system's working speed at $8000 \mathrm{r} / \mathrm{min}$, and analyze the influence law on the sealed rotor system with crack damage by changing the sealing gap of the system. The sealing gap varies from $0.05 \mathrm{~mm}$ to $0.17 \mathrm{~mm}$. Fig. 16 is the response diagram of the system with the change of the sealing gap. From the bifurcation diagram Fig. 16(a), it can be found that the airflow excitation state is before $0.126 \mathrm{~mm}$. The system is in pseudo-periodic motion, but the period window appears from $0.087 \mathrm{~mm}$ to 0.093 . After $0.126 \mathrm{~mm}$, the system enters a stable cycle-motion state. From the three-dimensional waterfall chart in Fig. 16(b), it can be found that the frequency of the system before $0.126 \mathrm{~mm}$ has $1 X, 2 X, f f, 2 f f$, and other combined frequencies, and there are two sudden changes in the excitation frequency within its range. In the Fig. 16(c) amplitude diagram of the system, it can be found that the amplitude of the system is also increasing with the increase of the sealing gap. When it is added to $0.123 \mathrm{~mm}$, it starts to drop sharply, and the amplitude reaches a stable state when the sealing gap is $0.126 \mathrm{~m}$.

(a)

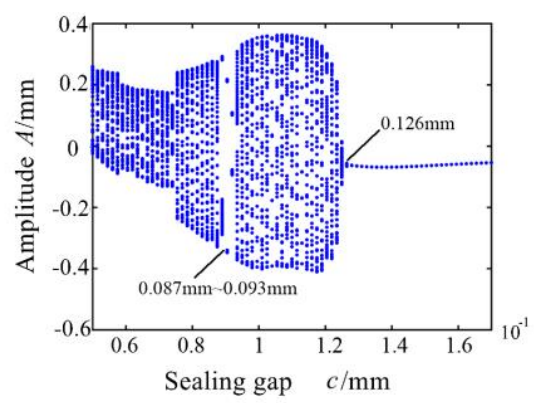

(b)

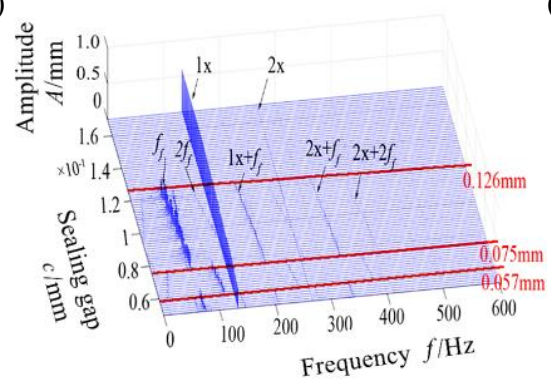

(c)

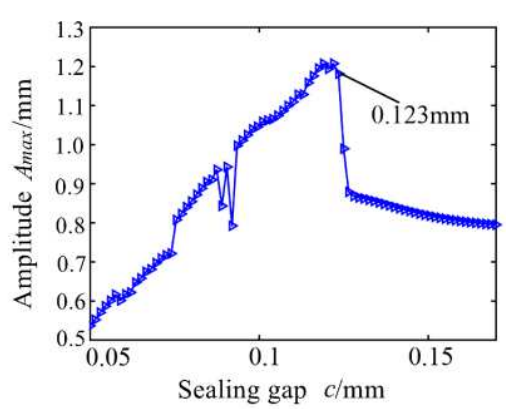

Fig. 16. The system response diagram with the change of the seal gap(a) Bifurcation diagram (b) $3 \mathrm{D}$ waterfall chart(c)Amplitude curve

\section{The effect of double crack damage on the seal-rotor system}

\subsection{Vibration characteristics of double crack damage}

This section analyzes the vibration characteristics of the seal-rotor system with double crack damage. When 
the position of the double crack is at the position of the 11-12 shaft section, the damage degree is $\mu=0.5$, and the system speed increases to 7000r/min. From the bifurcation diagram in Fig. 17(a), it can be seen that the speed does cycle one motion before $5500 \mathrm{r} / \mathrm{min}$, and then enters the pseudo-period state for a short period of time, and then does cycle one motion again at the end of 5700r/min. The $6200 \mathrm{r} / \mathrm{min}$ system once again Enter the pseudo-periodic movement. From Fig. 17(b), the frequency components of the system mainly include $1 X, 2 X, 3 X, 4 X$, ff and other combined frequencies. Compared with a single crack failure, the frequency doubling component caused by the crack is more obvious, and the frequency is high. If there are more components, the system will enter the unstable motion early, and the motion state in the high-speed zone will become complicated.

(a)

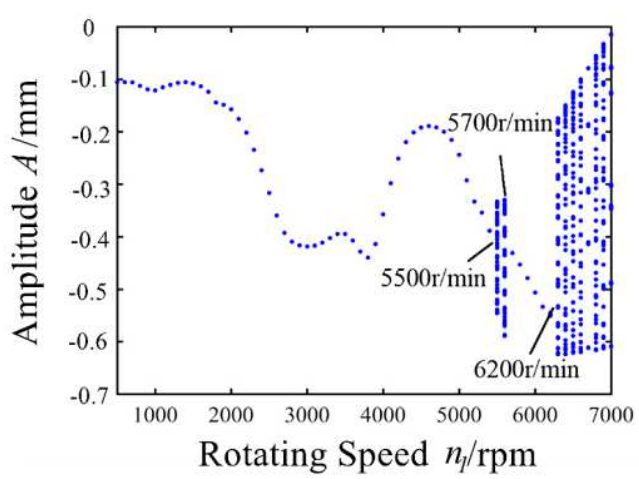

(b)

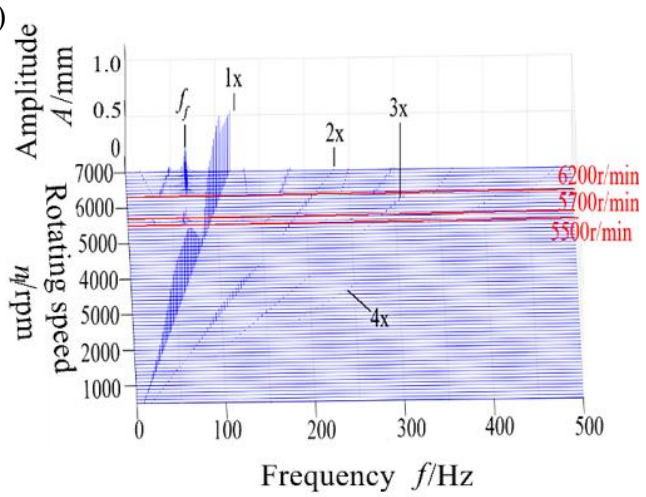

Fig. 17.The response characteristics of the double-crack fault seal-rotor system under the speed-up state(a)

Bifurcation diagram (b) 3D waterfall chart

\subsection{The effect of double crack damage location on system vibration characteristics}

This section considers the positions of the double cracks between nodes 10 and 13 in Fig. 1, so this section selects three representative cases: the double cracks are at nodes 11-13, 12-13, and 11- 12.Fig. 18 shows the system's axial trajectory and spectrogram when the system is at 1200r/min (around 1/2 times the critical speed) with different crack damage positions. The damage degree of the two cracks is $\mu=0.5$ (crack depth ratio). Fig. $18 \mathrm{~s}(\mathrm{a} 1)-(\mathrm{a} 2)$ shows the response characteristics of the cracks at nodes 11-13. The $2 \mathrm{X}$ raising system of the system is still based on power frequency. When the positions of the two cracks are at nodes 12-13 and 11-12, the distance between the two cracks becomes smaller. At this time, it can be seen from Fig. 18s(b1)-(b2)and(c1)-(c2) that the frequency of the system is mainly $2 X$ and $1 X$ is the auxiliary, and the system has super-resonance. It can be found in the axis trajectory diagram of the system that the axis trajectory of the system gradually moves in the direction of the arrow when the positions of the double cracks are close, and the axis trajectory changes from simple to complex. When double cracks occur, the axis track of the system is a figure-eight-like complex shape, which indicates that the damage position of the double cracks has a great influence on the vibration characteristics of the system. 


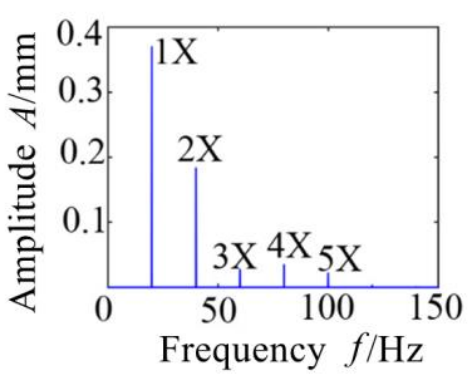

(a1)FFT spectrum

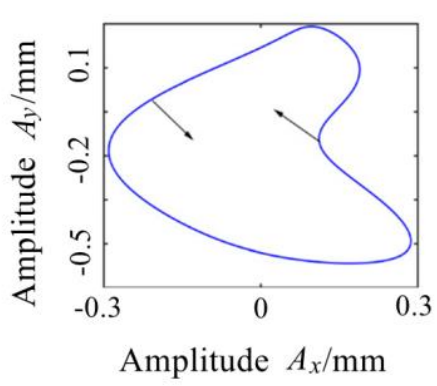

(a2)Axis trajectory diagram

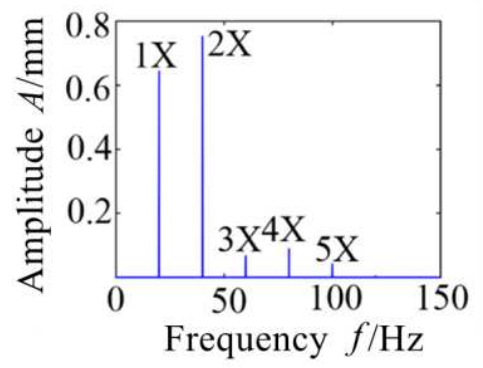

(b1)FFT spectrum

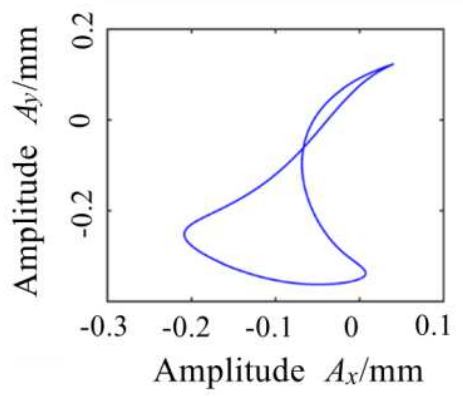

(b2)Axis trajectory diagram

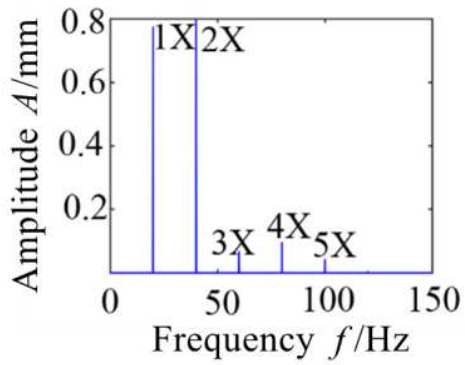

(c1)FFT spectrum

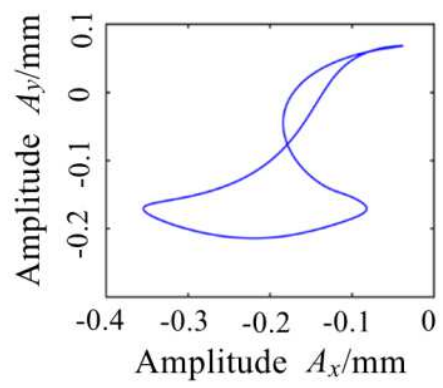

(c2)Axis trajectory diagram

Fig. 18. Vibration response diagram of the system with different crack positions at $1200 \mathrm{rev} / \mathrm{min}$ (a)11-13(b)12-13(c)11-12

5.3 The effect of the phase difference angle of the double crack on the vibration characteristics of the system

(a)

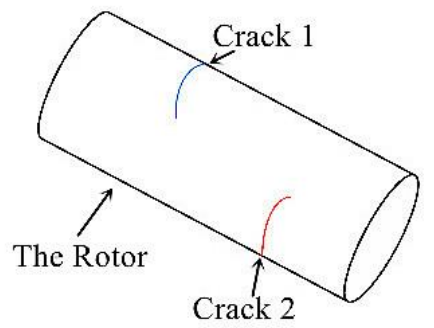

(b)

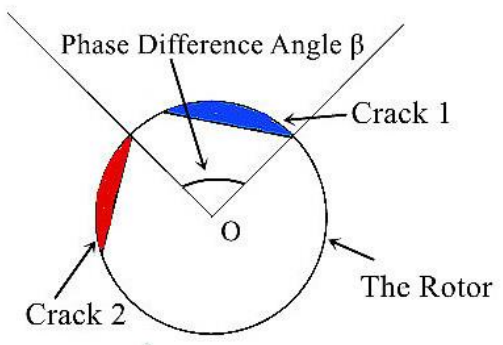

Fig. 19. Schematic diagram of crack angle difference

A schematic diagram of the crack angle difference is shown in Fig.19. The damage position of the double crack is selected as the node $12-13$, and the crack depth ratio of the double crack is $\mu=0.5$, and the system speed is set to $1200 \mathrm{r} / \mathrm{min}$. The change of the different angles of the double cracks is mainly by keeping the direction angle of the No. 13 node crack unchanged, and the direction angle of the No. 12 unit crack is changed in turn according to $\pi / 3, \pi / 2$, and $\pi$. From Fig. 20(a), it can be concluded that when the phase difference angle is slight (less than $\pi / 3$ ), the super-harmonic vibration of the system in the subcritical speed state is excited by the effect of the double cracks. The amplitude of the system $1 X$ and $2 X$ is increased. The value enhances the non-linear characteristics of the system and shows rich high-order harmonic components in the system's spectrogram and axis trajectory diagram. From Figure Fig. 20s(b1)-(b2)and(c1)-(c2), it can be seen that when the difference angle is large (greater than $\pi / 3$ ), 
the double cracks will inhibit each other as the difference angle increases. The power frequency component of the system will gradually increase and dominate. The super-harmonic vibration of the system disappears at the subcritical speed.

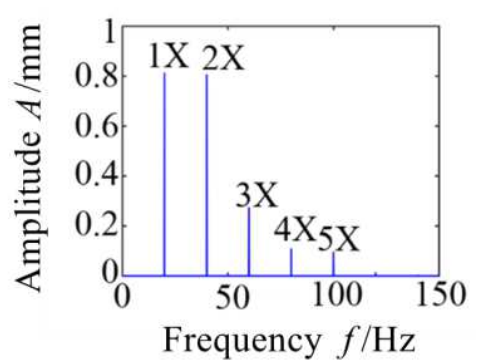

(a1)FFT spectrum

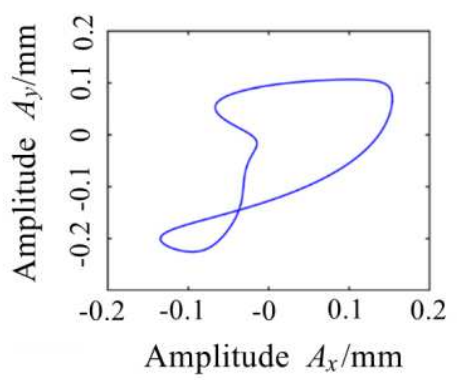

(a2)Axis trajectory diagram

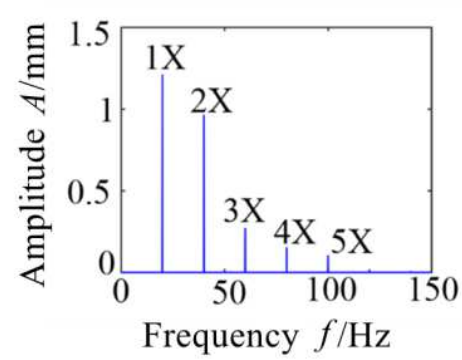

(b1)FFT spectrum

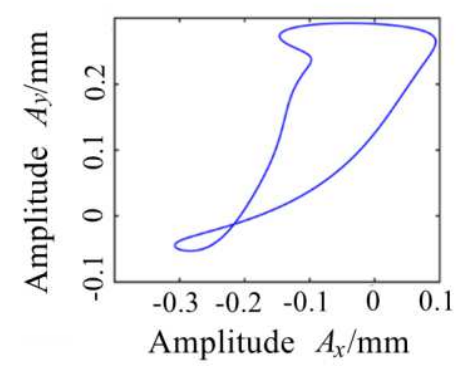

(b2)Axis trajectory diagram

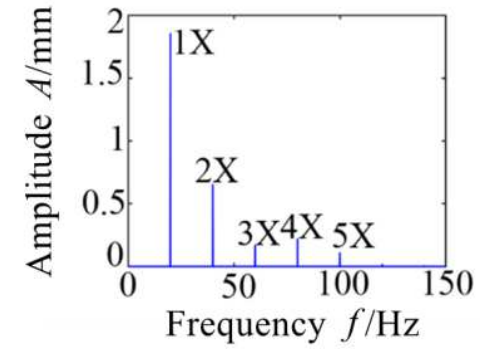

(c1)FFT spectrum

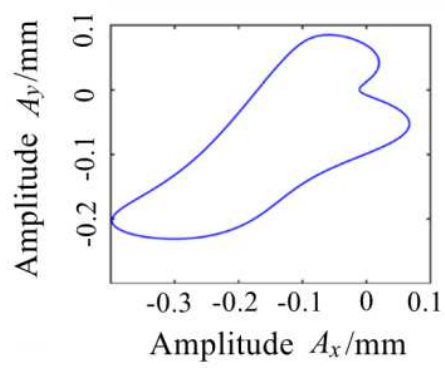

(c2)Axis trajectory diagram

Fig. 20. Vibration response diagram of $1200 \mathrm{r} / \mathrm{min}$ system with different phase difference angles(a) $\pi / 3$ (b) $\pi / 2$ (c) $\pi$

The influence of the different angle of the cracks on the frequency ff of the airflow excitation force generated after the instability of the system is further analyzed, and the damage degree of the double cracks $\mu=0.8$, the different angles are respectively $\pi / 4, \pi / 2$, and $\pi 3 / 4$, and the position is $12-13$ node Fig. 21 shows the frequency spectrum of the system under different crack angles when the system speed is $7000 \mathrm{rev} / \mathrm{min}$. In the figure, it can be found that when there is no difference angle, the airflow excitation frequency is $63 \mathrm{hz}$, and the amplitude is $0.13 \mathrm{~mm}$. When the difference angle increases to $\pi 3 / 4$, the ff amplitude of the system advances to $0.21 \mathrm{~mm}$. In summary, the crack difference angle has a nonlinear effect on the amplitude of the airflow excitation, and the increase of the crack difference angle will increase the amplitude of the airflow excitation frequency.

(a)

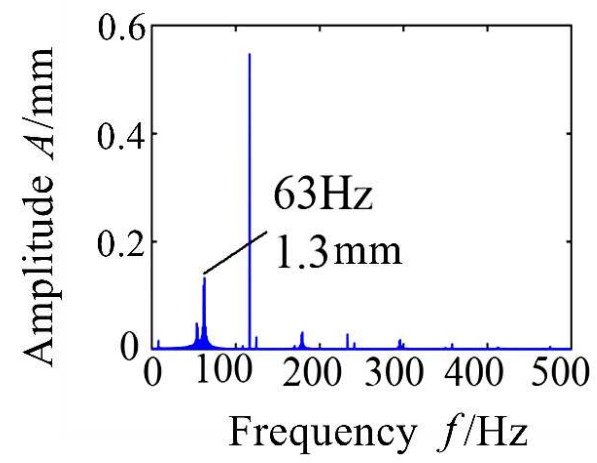

(b)

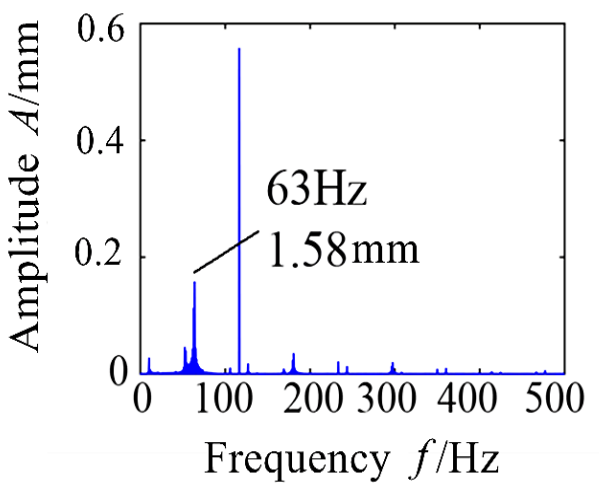


(c)

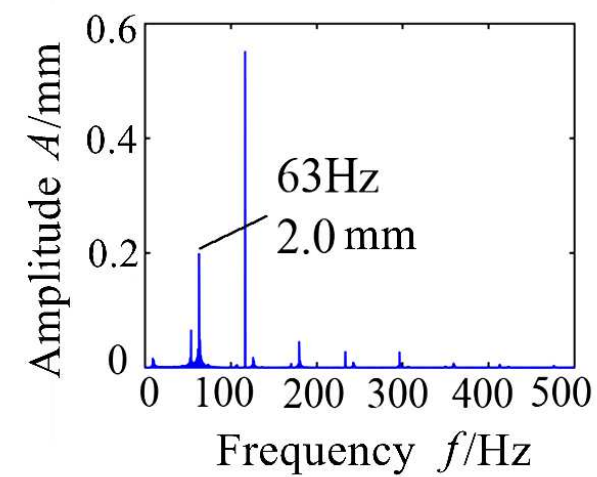

(d)

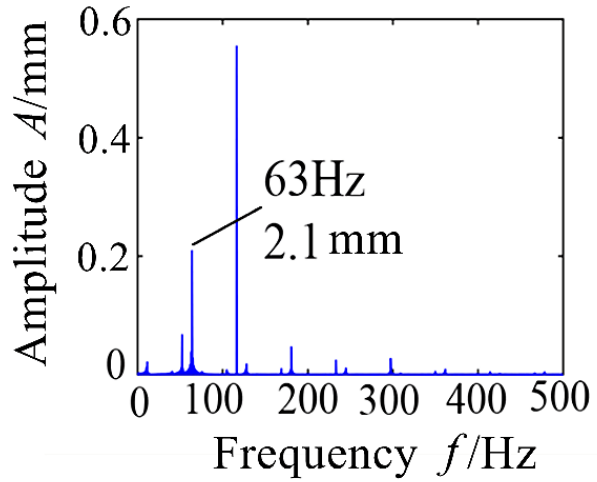

Fig. 21.Spectrogram of $7000 \mathrm{rev} / \mathrm{min}$ different crack angle system(a) $0 、(b) \pi / 4 、(c) \pi / 2 、(d) \pi 3 / 4$

\subsection{The effect of double crack damage on system vibration characteristics}

Based on the original parameters, the influence of the damage degree of the double crack on the vibration characteristics of the system under the subcritical speed state is further analyzed. The main analysis is that the phase difference angle of the double crack is 0 , and the damage degree of the double crack at the crack damage position is 12-13. Without changing the crack damage depth of No. 13 unit, its depth ratio $\mu=0.5$, change the crack damage depth of No. 12 unit. Fig. 22 shows the axis trajectory and frequency spectrum of the system in the subcritical speed region when the crack damage degree $\mu$ is $0.3,0.4$, and 0.5 , respectively. As the crack depth increases, it can be found that the amplitude of the power frequency of the system is gradually decreasing, and the amplitude of the high frequency of the system is gradually increasing. It can be found that the frequency of the system is dominated by the power frequency when the crack depth ratio is less than 0.5 . High multiplier frequency is supplemented, no superharmonic vibration occurs. When the crack depth is 0.5 , the $2 X$ of the system exceeds the power frequency, and $2 X$ begins to dominate, and the system begins to generate superharmonic vibration. With the increase of crack depth, the amplitude of $2 X$ and other high-frequency components is increasing, the amplitude of power frequency is decreasing, and the axis trajectory of the system is constantly becoming more complicated.

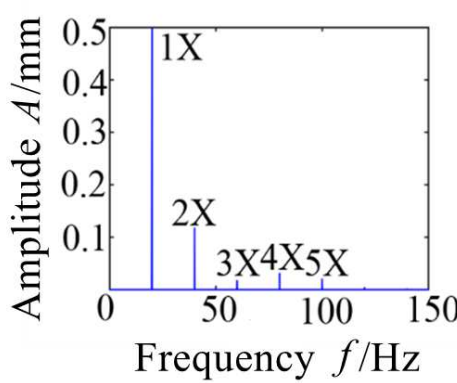

(a1)FFT spectrum

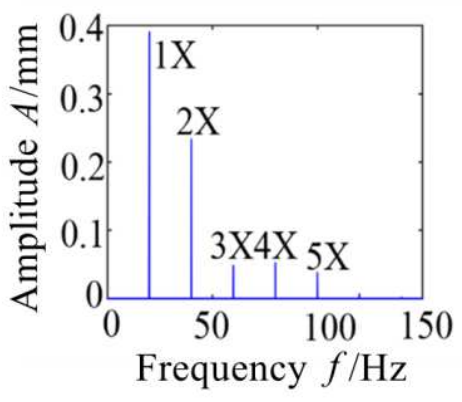

(b1)FFT spectrum

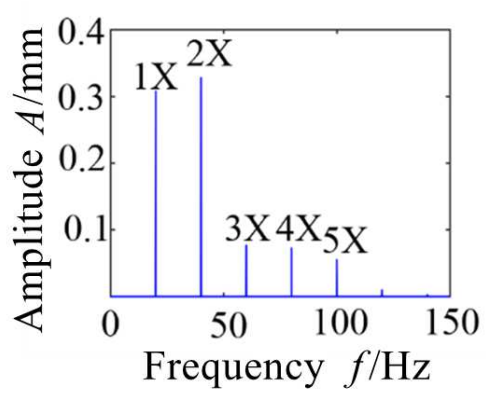

(c1)FFT spectrum 


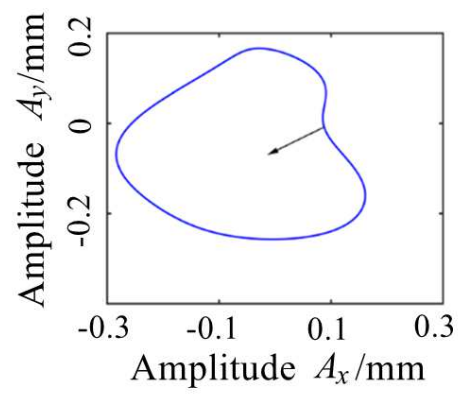

(a2)Axis trajectory diagram

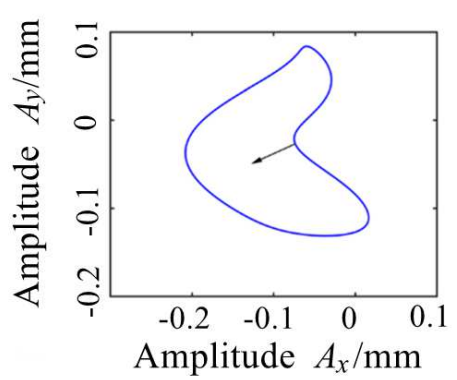

(b2)Axis trajectory diagram

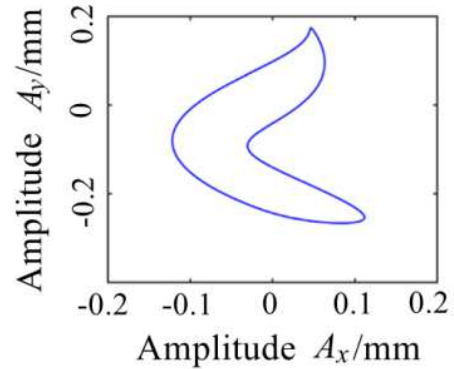

(c2)Axis trajectory diagram

Fig. 22.System response diagram of $1200 \mathrm{r} /$ min double cracks with different damage levels(a) $\mu=0.3$ (b) $\mu=0.4$ (c)

$$
\mu=0.5
$$

\subsection{Experimental analysis of double crack damage rotor system}

The double crack damage test mainly analyzes the system's response characteristics when the depth of one of the cracks is constantly changing. The main parameters refer to the damage parameters of the double crack in Section 5.4. The damage location of the double cracks in the shaft section 12-13, The different angle of the double crack is 0 degrees, and the system speed is $1270 \mathrm{r} / \mathrm{min}$ (near the $1 / 2$-order critical speed), and the crack damage degree of the No. 12 shaft section is also kept unchanged. As shown in Fig. 23, the crack damage degree of the No. 13 shaft section $\mu$ is $0.3,0.4,0.5$.

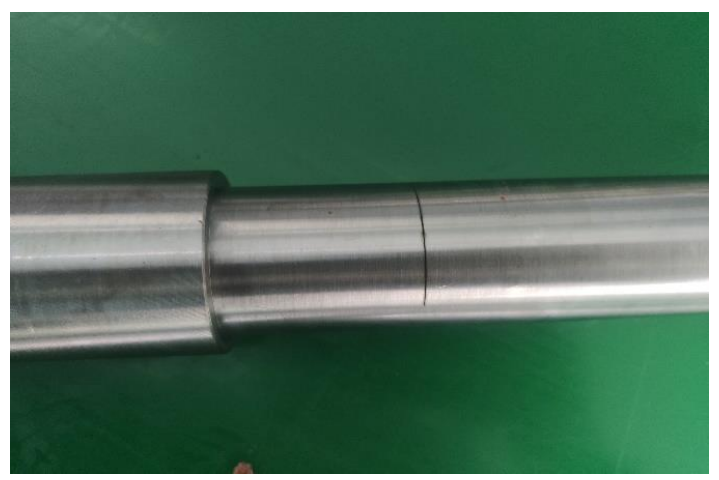

Fig. 23. Crack damage diagram of No. 13 node of the test bench

Fig.24 is the response diagram of the vibration experiment of the system with different phase difference angles of $1200 \mathrm{r} / \mathrm{min}$. It can be seen from the experimental result diagram that it corresponds exactly to the simulation result of Fig. 20. From Fig. 24s(a1)-(a2), when the phase difference angle is $\pi / 3$, the super-harmonic vibration of the system in the subcritical speed state is stimulated by the action of the double cracks, which shows a lot in the system's spectrogram and axis trajectory graph. The high-order harmonic components; From Fig. $24 \mathrm{~s}(\mathrm{~b} 1)-(\mathrm{b} 2)$ and(c1)-(c2), when the phase difference angle is large, the double cracks inhibit each other, which makes the power frequency component of the system gradually increase and dominate, and the super-harmonic vibration of the system disappears at the subcritical speed. 


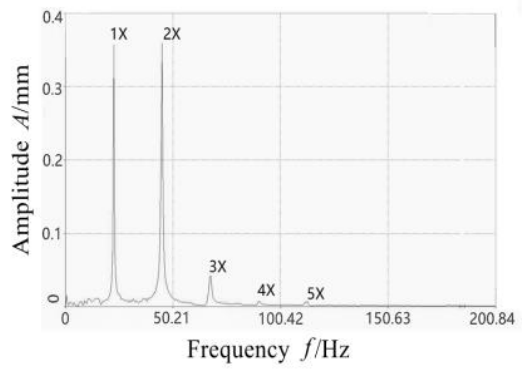

(a1)FFT spectrum

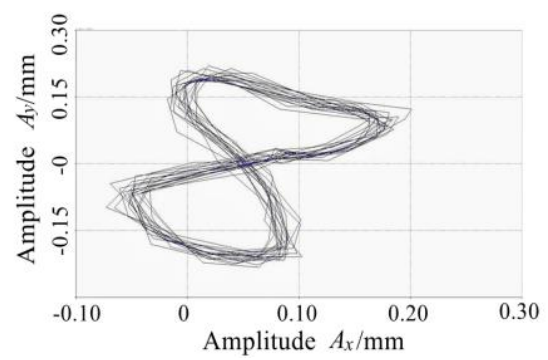

(a2)Axis trajectory diagram

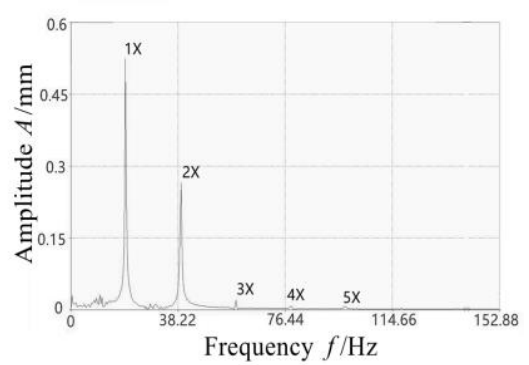

(b1)FFT spectrum

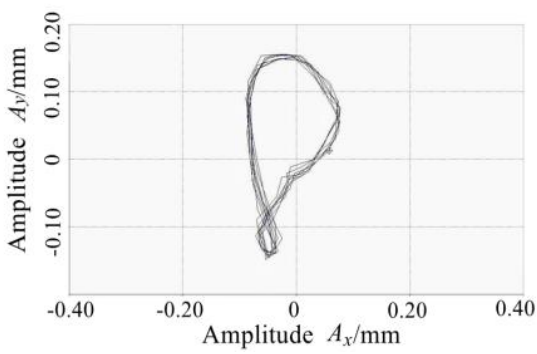

(b2)Axis trajectory diagram

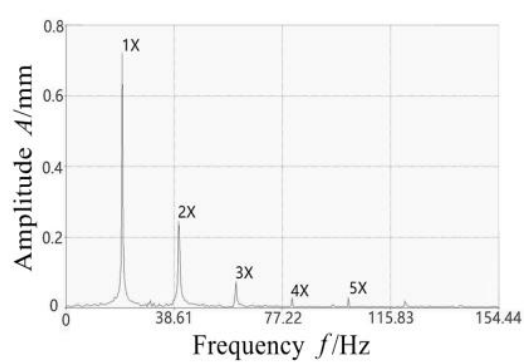

(c1)FFT spectrum

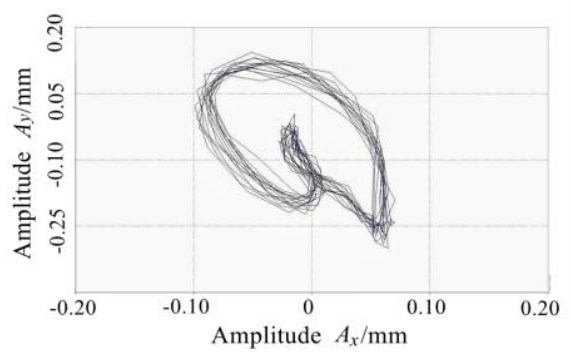

(c2)Axis trajectory diagram

Fig. 24.Vibration experiment response diagram of 1200r/min double crack system with different phase difference

$$
\text { angles(a) } \pi / 3(b) \pi / 2 \text { (c) } \pi
$$

Fig. 25 shows the experimental spectrum of the system with different crack angles at $7000 \mathrm{r} / \mathrm{min}$ and the crack depth $\mu=0.5$. It can be seen from the test result that it is consistent with the simulation result in Fig.21. In the figure, it can be found that the airflow excitation frequency is $63 \mathrm{hz}$ when there is no difference angle. As the phase difference angle increases, the amplitude increases from $0.35 \mathrm{~mm}$ to $0.80 \mathrm{~mm}$.In summary, the crack difference angle has a nonlinear effect on the amplitude of the airflow excitation. The increase of the crack difference angle will increase the amplitude of the airflow excitation frequency, indicating the accuracy of the simulation results.

(a)

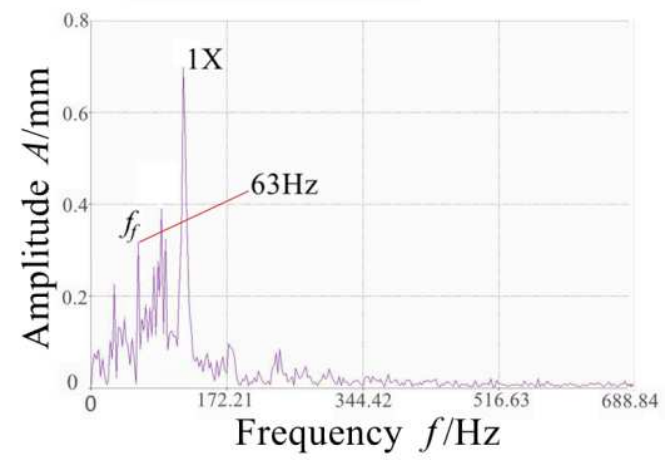

(b)

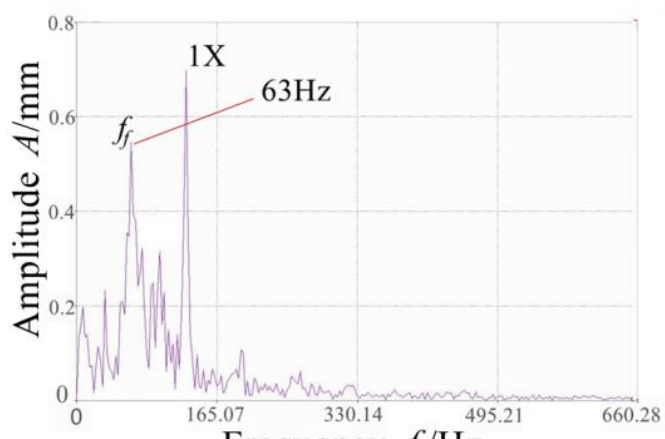

(c)

Frequency $f / \mathrm{Hz}$ 
(d)
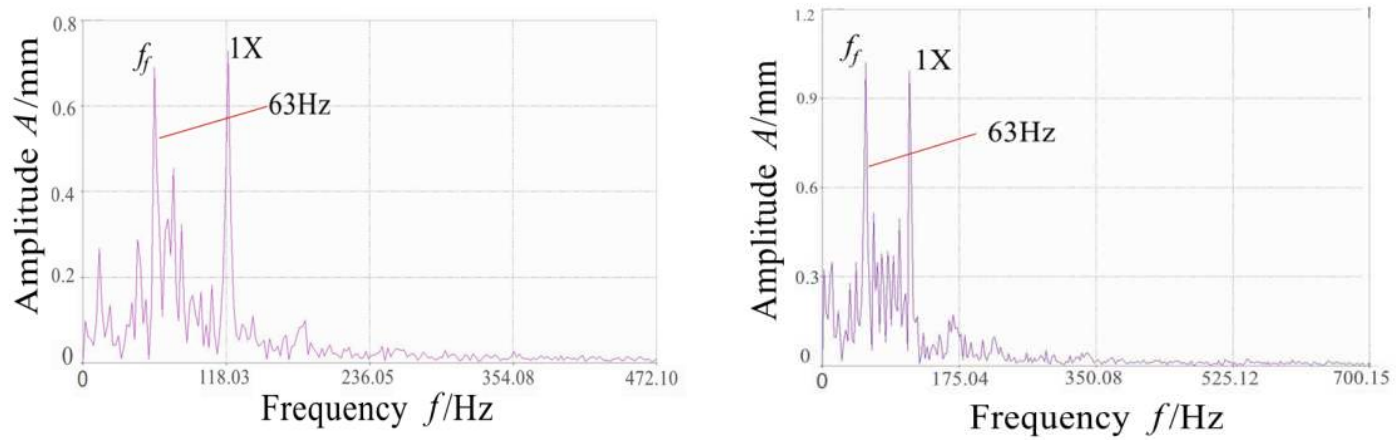

Fig. 25.7000r/min Different Crack Angle System Experimental Spectrogram(a)0 (b) $\pi / 4(c) \pi / 2(d) \pi 3 / 4$

Fig.26 shows the response diagram of the system under different levels of damage. In the spectrogram of the system, it can be found that as the degree of crack damage increases, the frequency doubling component of the system gradually becomes obvious $2 X, 3 X$ and other frequency doubling phenomena. And the amplitude of $2 \mathrm{X}$ is gradually increasing. When the damage degree increases to a particular value, the $2 X$ component of the system exceeds the power frequency and dominates. The phenomenon is consistent with the simulation results. The axis trajectory is also the same as the simulation results. Phenomenon.

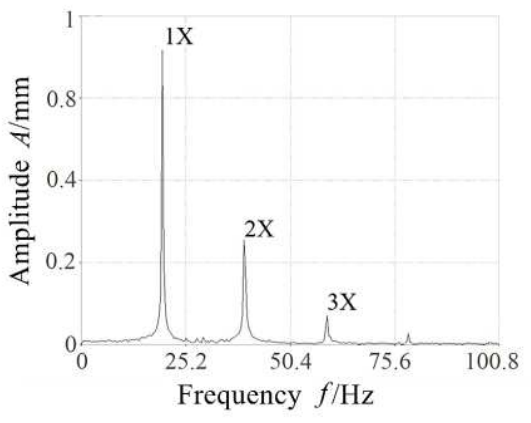

(a1)FFT spectrum

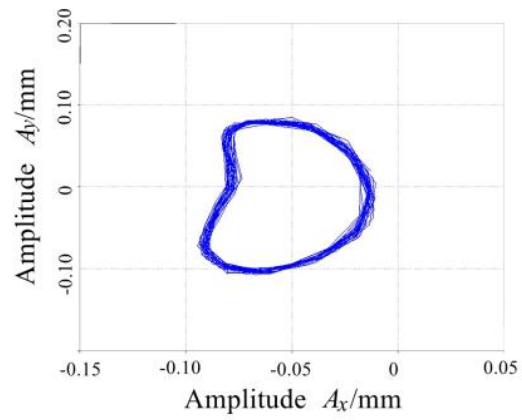

(a2)Axis trajectory diagram

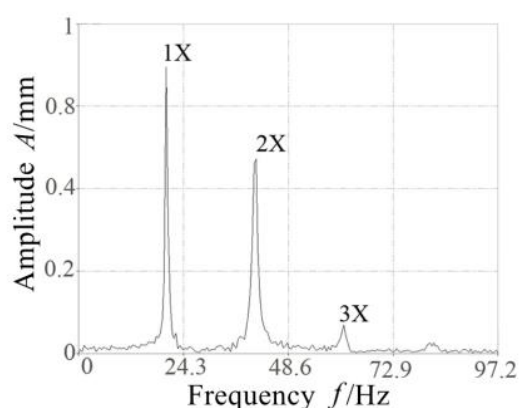

(b1)FFT spectrum

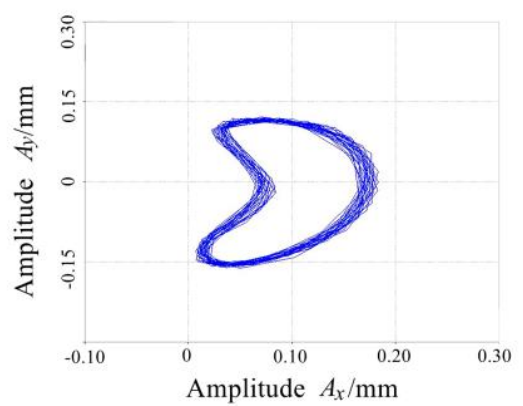

(b2)Axis trajectory diagram

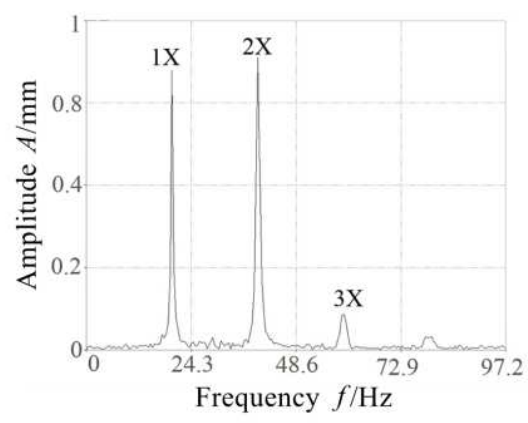

(c1)FFT spectrum

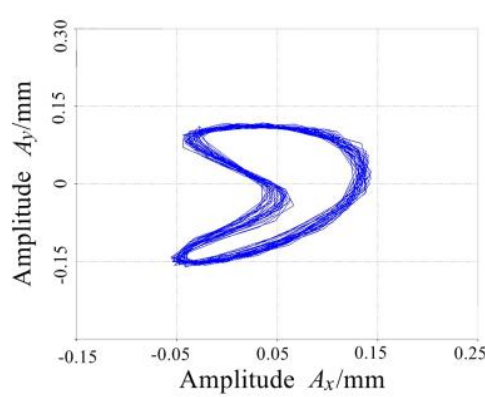

(c2)Axis trajectory diagram

Fig. 26. Response graph of system test with different loss degree of node 12 (a) $\mu=0.3$ (b) $\mu=0.4$ (c) $\mu=0.5$

\section{Conclusion}

Based on the labyrinth seal-rotor system, this paper simulates the crack damage failure of the rotating shaft and considers the influence of the gyro effect and the sealing force. Based on the finite element theory, the dynamic 
equation of the system is established, and the calculation is solved by the Newmark- $\beta$ numerical integration method. The vibration displacement data of the system draws three-dimensional waterfall diagrams, bifurcation diagrams, time-domain waveform diagrams, axis trajectory diagrams, spectrogram diagrams and Poincaré cross-section diagrams to analyze the crack damage in the seal-rotor system and the impact of crack damage on the system when the sealing parameters are changed. The conclusions obtained are as follows:

(1)When there is no sealing force in the system, the crack damage failure causes the first-order critical speed of the system to decrease and the resonance amplitude to increase, and this feature becomes more evident with the increase of the crack damage degree and it also induces superharmonic resonance in the subcritical region. Phenomenon; After the sealing force is stored in the system, the airflow force in the system will weaken the high-frequency resonance characteristics caused by cracks in the subcritical speed zone so that the amplitude of the system is reduced and the range of axis trajectory is reduced; As the crack deepens, the crack will increase the amplitude of the system and affect the instability speed of the rotor system. The dynamic characteristics of the system in the super first-order critical speed region are mainly characterized by airflow excitation, but with the degree of crack damage Increase, the $f f$ frequency and amplitude of the system will gradually decrease.

(2)When there is a sealing force: increasing the sealing pressure difference, the sealing gap, and the sealing length will increase the instability speed of the system, delay the occurrence of airflow excitation, and make the movement state of the system in the high-speed region more complicated. With the increase of seal pressure difference, length, and gap, the range of pseudo-periodical motion gradually decreases. When it increases to a certain value, the system always maintains a period-one motion state. It is found that a large sealing gap can weaken the high-frequency characteristics of the system caused by cracks, but when the sealing gap is large, the leakage of the system fluid will be increased, and the sealing effect of the system will be affected.

(3) The influence law of sealing parameters on system airflow excitation: In the research of keeping the system speed constant and changing the sealing pressure difference, length, and gap of the system, it is found that increasing the sealing pressure difference will gradually reduce the frequency and amplitude of the excitation generated after the system is unstable; Increasing the length of the seal will reduce the frequency and amplitude of the system's airflow excitation, but a sudden change will occur during it; increasing the sealing gap will induce two sudden changes in the airflow excitation within a smaller range, which shows that the sealing parameters affect the system. Have a greater impact.

(4)The effect of double crack failure on the seal-rotor system: Compared with single-crack failure, double-crack will increase the amplitude of the frequency-doubling component of the system and will also increase the high-frequency component such as $4 X$. Double-crack failure will advance the instability speed of the system; Under the action of double cracks, the nonlinear characteristics of the system will be increased. When the phase 
angle is large, the power frequency of the system exceeds $2 X$ dominates, and the crack difference angle can enhance the influence of the crack failures to cancel each other. Increasing the damage depth of the crack will gradually reduce the $1 X$ amplitude of the system, and the frequency amplitude of $2 X$ and other high-power components will gradually increase. When the crack depth is significant, the system will experience subcritical speed super-harmonic vibration.

\section{Acknowledgment}

The project supported by the Major Program of the National Natural Science Foundation of China (Grant No.51875085), and the Natural Science Foundation of Liaoning Province, China (Grant No.20180551073).

\section{data availability statement}

The raw/processed data required to reproduce these findings cannot be shared at this time as the data also forms part of an ongoing study.

\section{Declaration of Competing Interest}

The authors declare that they have no known competing financial interests or personal relationships that could have appeared to influence the work reported in this paper.

\section{References}

[1] Y.G. Luo, Review and prospect on the research of the labyrinth seal-rotor system , Journal of Dalian Minzu University, 21.01(2019):6-14. http//doi:10.13744/j.cnki.cn21-1431/g4.2019.01.002.

[2] Muszynska A. Rotor to stationary element rub-related vibration phenomena in rotating machinery-literature survey, Shock and Vibration Digest, 21.3(1989):3-11.

[3] W.Z. Wang, Y.Z. Liu, Numercial analysis of leakage flow through two labyrinth seals , Journal of hydrodynamics, 01(2007):107-112. http//doi:CNKI:SUN:SDYW.0.2007-01-017.

[4] F. Cangioli, et al. Effect of energy equation in one control-volume bulk-flow model for the prediction of labyrinth seal dynamic coefficients, Tribology international, Mechanical Systems and Signal Processing 98.1(2018):594-612. http//doi:10.1016/j.ymssp.2017.05.017

[5] R. G. Kirk , Z. Guo . Influence of leak path friction on labyrinth seal inlet swirl , Tribology Society of Tribologists and Lubrication Engineers, 52(2009):139-145.

[6] T. Hirano, Z Guo, R.G. Kirk ,Application of computational fluid dynamics analysis for rotating machinery: part II- labyrinth seal analysis, Asme Turbo Expo, Collocated with the International Joint Power Generation Conference 2003.

[7] C. Avza, C. Yblb , Computational analysis for scallop seals with sickle grooves, part II: Rotordynamic characteristics.Mechanical Systems and Signal Processing 147.http//doi:10.1016/j.ymssp.2020.107154

[8] S.Subramanian, A. S.Sekhar, B. V. S. S .S. Prasad . Rotordynamic characteristics of rotating labyrinth gas $\begin{array}{lllll}\text { turbine } & \text { seal } \quad \text { with } \quad \text { centrifugal Trowth }\end{array}$ International,97(2016):349-359.http//doi:10.1016/j.triboint.2016.01.003 
[9] D. Sun ,S. Wang , Y.T. Ai , et al. Theoretical and experimental research on the performance of anti-swirl flow for the static and dynamic characteristics of seals , Journal of mechanical engineering, 52.3(2016):101.http//doi:10.3901/JME.2016.03.101

[10] LIU Xingwang, ZHANG Guanzheng, LI Chao, et al. Research on gas leakage pneumatic thermodynamic behavior in the radial labyrinth of scroll compressor, Journal of mechanical engineering, 51.20(2015):201.http//doi:10.3901/JME.2015.20.201

[11] W.S. Ma ,H. Huang, G. Q. Feng, et al. Labyrinth seals diameter and length effect study on nonlinear dynamics , Procedia engineering, 99(2015):1358-1364.http//doi:10.1016/j.proeng.2014.12.670

[12] S. H. Shyu, Y. W. Chen, Dynamic characteristics of rotor-bearing system with a labyrinth seal ,Key engineering materials, 739(2017):169-181.http//doi:10.4028/www.scientific.net/KEM.739.169

[13] Rao X B , Chu Y D , Chang Y X , et al. Dynamics of a cracked rotor system with oil-film force in parameter space,Nonlinear Dynamics, 2017, 88(4):2347-2357.http//doi:10.1007/s11071-017-3381-9

[14] A. K. Darpe ,K. Gupta , A. Chawla , Transient response and breathing behaviour of a cracked Jeffcott rotor , Journal of Sound and Vibration,272.1-2(2004):207-243.http//doi:10.1016/S0022-460X(03)00327-4

[15] A. K. Darpe , Dynamics of a Jeffcott rotor with slant crack, Journal of Sound and Vibration, 303.1-2(2007):1-28.http//doi:10.1016/j.jsv.2006.07.052

[16] T. H. Patel , M. J. Zuo , A. K. Darpe , Vibration response of misaligned rotors, Journal of Sound and Vibration 325. 3(2009):609-628.http//doi:10.1016/j.jsv.2009.03.024

[17] T. H. Patela , A. K. Darpe , Influence of crack breathing model on nonlinear dynamics of a cracked rotor, Journal of Sound and Vibration, 311.3-5(2008):953-972.http//doi:10.1016/j.jsv.2007.09.033

[18] T. Szolc , P. Tauzowski, Damage identification in vibrating rotor-shaft systems by efficient sampling approach, Mechanical Systems and Signal Processing, 23.5(2009):1615-1633.http//doi:10.1016/j.ymssp.2008.12.007

[19] Lu .Z , Hou .L, Chen .Y, et al. Nonlinear response analysis for a dual-rotor system with a breathing transverse crack in the hollow shaft, Nonlinear Dynamics, 2016, 83(1-2):169-185.http//doi:10.1007/s11071-015-2317-5

[20] Q. Han , F. Chu ,The effect of transverse crack upon parametric instability of a rotor-bearing system with an asymmetric disk, Communications in Nonlinear Science and Numerical Simulation, 17.12(2012):5189-5200.http//doi:10.1016/j.cnsns.2012.03.037

[21] Q. K. Han , J. S. Zhao , F. L. Chu , dynamic analysis of a geared rotor system considering a slant crack on the shaft, journal of Sound and Vibration, 331.26(2012):5803-5823.http//doi:10.1016/j.jsv.2012.07.037

[22] Palacios-Pineda, L.M, Gomez-Mancilla, J.C , et al. The influence of a transversal crack on rotor nonlinear transient response,Nonlinear Dynamics,2017,90(1):671-682.http//doi:10.1007/s11071-017-3687-7

[23] Y. G. Luo , B. C.Wen . Nonlinear response of a double-span rotor system with crack-loose coupling fault, Journal of Aeronautics and Dynamics, 26.3(2005):289-292.http//doi:10.1360/biodiv.050084

[24] Y. F.Yang, X. M. Ren, W. Y. Qin, Nonlinear response of a loose-crack coupled fault rotor system, Mechanical Science and Technology, 24.8(2005):3.http//doi:10.3321/j.issn:1003-8728.2005.08.029

[25] Xiang L , Deng Z , Hu A, et al. Multi-fault coupling study of a rotor system in experimental and numerical analyses,Nonlinear Dynamics, 2019, 97(4):2607-2625.http//doi:10.1007/s11071-019-05151-x

[26] H. Ma , B. C. Wen , X. Y. Tai , et al. Dynamics of rotating blade-casing systems with rubbing, Beijing: The Science Publishing Company, 2017. 
[27] A. Muszynska, D. E. Bently, Chaotic responses of unbalanced rotor/bearing/stator systems with looseness or rubs, Journal of sound \& vibration, 5.9(1995):1683-1704.http//doi:10.1016/0960-0779(94)00171-L

[28] H. Ma, H. Li , H. Q. Niu , et al. Nonlinear dynamic analysis of a rotor-bearing-seal system under two loading conditions , Journal of sound \& vibration, 332.23(2013):6128-6154.http//doi:10.1016/j.jsv.2013.05.014

[29] Y. Luo , S. Zhang, B. Wu , et al. Dynamic Analysis on Nonlinear Fluid-Structure Interaction Forces of Rub-Impact Rotor System [J]. Open $\quad$ Mechanical Engineering Journal,8.1(2014):480-486.http//doi:10.2174/1874155X01408010480

[30] S. T. Li , Q. Y. Xu , X. L. Zhang . Nonlinear dynamic behaviors of a rotor-labyrinth seal system [J]. Nonlinear Dynamics, 47.4(2006):321-329.http//doi:10.1007/s11071-006-9025-0 